\title{
Die Österreichische Mindestsicherungsreform 2010: Von der Armuts- zur Arbeitsmarktpolitik
}

\author{
Marcel Fink ${ }^{1, *}{ }^{*}$, Bettina Leibetseder ${ }^{2,{ }^{\star \star}}$ \\ 1 Institut für Höhere Studien (IHS) Wien \\ 2 HAW Landshut, Landshut \\ * fink@ihs.ac.at, ** bettina.leibetseder@haw-landshut.de
}

\section{Zusammenfassung}

Gegen den europäischen Mainstream war die originäre Zielsetzung der in den 20ooer Jahren in Österreich verhandelten Sozialhilfereform eine einheitliche und armutsfeste Leistung zu schaffen. Die inhaltliche Ausgestaltung der im Jahr 2010 beschlossenen Bedarfsorientierten Mindestsicherung (BMS) erfüllte diese Bestrebungen jedoch schlussendlich nicht. Der Beitrag analysiert den gegenständlichen Prozess ausgehend vom bis in die I980er Jahre zurückgehenden und sich wiederholt wandelnden Agendasetting bis zum Beschluss im Nationalrat im Jahr 20IO. Als zentral kristallisiert sich dabei, vor dem Hintergrund unterschiedlicher theoretischer Erklärungsansätze, die sich mehrfach verändernde strategische und inhaltliche Positionierung der Sozialdemokratischen Partei (SPÖ) heraus. Ungeachtet dessen, dass ein Eintreten für vergleichsweise generöse Mindestsicherungsleistungen bei politischen AkteurInnen, die ein breites WählerInnenspektrum anzusprechen versuchen, generell eher unwahrscheinlich ist, wird am österreichischen Fall ersichtlich, dass sozialdemokratische Parteien - unter bestimmten „günstigen“ Rahmenbedingungen und mobilisiert von individuellen AkteurInnen - auch proaktiv die Interessen von (potentiellen) MindestsicherungsbezieherInnen und damit von so genannten „Outsidern“vertreten. Ändert sich der Kontext jedoch in eine Richtung, die ein solches Engagement aus strategischen Überlegungen heraus fragwürdig erscheinen lässt, so besteht eine hohe Wahrscheinlichkeit, vom ursprünglichen Kurs bzw. der früheren Argumentationsbasis abzurücken. Letzteres erleichtert es anderen politischen Kräften wiederum die gegenständliche Debatte dominant in einen gänzlich anderen inhaltlichen Rahmen, im gegenständlichen Fall jenen von „Sozialmissbrauch und Leistungsgerechtigkeit“, zu verschieben.

\section{Schlüsselwörter}

Sozialhilfe, Mindestsicherung, Österreich, Parteiendifferenzhypothese, SPÖ

\section{The 2010 Reform of the Minimum Income Scheme in Austria: From Poverty Alleviation to Labour Market Policy}

\begin{abstract}
Against the European tide, the original objective of a social assistance reform, negotiated in Austria as from the 20oos, was to create a uniform and poverty-proof benefit. However, the Means-tested Minimum Income Scheme (MMS) adopted in 2010 did not fulfil these aims. This article discusses the challenges and factors that transformed the reform from the agenda setting in the I980s to the adoption of the legislation in the parliament in 20IO. Against the backdrop of various explanations, the strategic position and problem-framing of the Austrian Social Democratic Party as the main actor, is pivotal. Political actors that attempt to address a wide range of voters are generally unlikely to advocate comparatively generous minimum benefits. However, the Austrian case exemplifies that Social Democrats can proactively represent the interest of (potential) minimum income recipients and thus of so-called 'outsiders' under specific favourable conditions and in case of the the mobilization of individual actors. Yet, whenever the context changes in a direction that makes such commitment questionable for strategic reasons, there is a high likelihood to move away from the original course and the related dominating reasoning. The latter, in turn, makes it easier for other political forces to entirely re-frame the debate - in the present case towards issues like "benefit fraud' and 'meritocracy'.
\end{abstract}

\section{Keywords}

Social Assistance, Minimum Income, Austria, Social Democrats, Hypothesis on Party Differences 


\section{Einleitung, Fragestellung und Methode}

Mindestsicherungssysteme standen in den hoch entwickelten Wohlfahrtsstaaten lange Zeit nicht im Fokus sozialpolitischer Auseinandersetzungen ${ }^{\mathrm{I}}$ und Analysen. Jedoch sehen sich Mindestsicherungssysteme seit den I980er Jahren international mit wachsenden Herausforderungen konfrontiert. Reformen der Systeme und vergleichende Forschung zu den Ausgestaltungsformen, Wirkungen und Entwicklungen der Mindestsicherungssysteme folgten (Gough et al. 1997; Bahle et al. 20II; Figari et al. 2013).

Die wachsende Nachfrage nach Leistungen der Mindestsicherungssysteme kann auf ein Bündel an Ursachen zurückgeführt werden. Aufgrund von struktureller Arbeitslosigkeit und der Postindustrialisierung der Arbeitsmärkte können insbesondere niedrig qualifizierte Personen dem Ziel kontinuierlicher Beschäftigung vermehrt nicht mehr entsprechen. Deren Erwerbseinkommen werden immer weniger „armutsfest". Des Weiteren veränderten sich die „üblichen“ Formen des familiären Zusammenlebens und es differenzierten sich die Erwerbschancen und -risiken auf Haushaltsebene. Alleinstehende und Alleinerziehende sind dadurch mit ungleich höheren sozialen Risiken und Problemen konfrontiert als Personen in - ebenfalls stärker verbreiteten - Haushalten mit mehreren Erwerbstätigen (Lødemel/ Moreira 20I4; Marx/Nelson 20I3). Die Interaktion zwischen diesen ökonomischen und soziodemografischen Trends bzw. ihr Zusammentreffen führte vielfach zu zunehmenden sozialen Problemen, die durch den "Kern“ der sozialen Sicherungssysteme, im gegenständlichen Zusammenhang insbesondere die Arbeitslosenversicherung, nicht oder nur unzureichend abgesichert sind. Insgesamt sind breitere Bevölkerungsgruppen von postindustriellen "neuen sozialen Risiken“ (Bonoli 2007; Taylor-Gooby 2004) betroffen, was sich in einem wachsenden Bedarf an residualen Leistungen manifestiert.

In den meisten Ländern wurden dabei die vorgelagerten Sozialversicherungssysteme nicht ausgebaut. Im Gegenteil, Reformen in der Arbeitslosenversicherung erschwerten ab den I990er Jahren vielfach den Leistungszugang, verkürzten die -dauer und/oder das -niveau. Dies stimulierte den Zustrom zu sozialen Mindestsicherungssystemen zusätzlich (Clasen/Clegg 2OII). Was die Systemausgestaltung betrifft, so ist in Bezug auf diesen Zweig sozialer Sicherung international kein expansiver Entwicklungspfad evident. Wie in vorgelagerten Leistungen und manchmal sogar darüber hi-

I Dies gilt jedenfalls für die - gemäß der Typologie von EspingAndersen (1990) - „sozialdemokratischen“ Sozialstaaten in Nordeuropa und die „konservativ-korporatistischen“ in Kontinentaleuropa. Im Gegensatz dazu wird die Rolle bedarfsgeprüfter Leistungen als traditionelles und definitorisches Merkmal des „liberalen“ Sozialstaatstypus angesehen. naus kam es vielfach zu einem Rückbau (Nelson 20I3). Zugleich wurden die Konditionalitäten für die Betroffenen schrittweise ausgebaut und über sogenannte Aktivierungsprogramme zunehmend an über soziale Bedürftigkeit hinausgehende Voraussetzungen gebunden (vgl. z.B. Clasen/Clegg 2012; Weishaupt 20II), was einen substanziellen Wandel hinsichtlich sozialer Rechte in Mindestsicherungssystemen einleitete (vgl. Bahle et al. 20II). Und auch der steigende Bedarf an Mindestsicherungsleistungen aufgrund der internationalen Finanzund Wirtschaftskrise veränderte diesen Trend sozialpolitischer Steuerung nicht (vgl. Marchal et al. 20I4).

Der Reform des österreichischen Mindestsicherungssystems, bei der die traditionelle "Sozialhilfe" ab Herbst 2010 durch die so genannte „Bedarfsorientierte Mindestsicherung" (BMS) abgelöst wurde, ging eine mehr als zehnjährige Debatte voraus (Pfeil/Otter 2OII). Im internationalen Vergleich stellt Österreich dabei einen abweichenden Fall dar. Zuerst standen nämlich primär armutspolitische Fragen im Fokus der gegenständlichen Reformdebatten (Tálos 2008a; Dimmel 2008). Es erfolgte keine sonst weit verbreitete (vgl. z.B. Clasen/ Clegg 20I2) Verengung auf die Arbeutsmarkteinbindung von LeistungsbezieherInnen. Erst in der letzten Phase des Reformprozesses kam es zu einer maßgeblichen Erosion von inhaltlichen Elementen, die ursprünglich entgegen dem europäischen Trend in einen breiten Ausbau sozialer Rechte gemündet hätten. Letzteres, obwohl ein für die gegenständliche Reform relevanter politischer Akteur - nämlich die Sozialdemokratische Partei Österreichs (SPÖ) - dies lange Zeit als fundamentale Reformbestrebung ventiliert hatte. Die zuerst dominierenden explizit armutspolitischen Ziele wurden schlussendlich hinter die arbeitsmarktpolitischen gestellt (vgl. Leibetseder/Woltran 20II).

Der vorliegende Beitrag geht erstens der Frage nach, warum und mit welcher Zielorientierung die Frage sozialer Mindestsicherung auf die Österreichische politische Agenda gesetzt wurde. Zweitens werden wesentliche Schritte und Einflussfaktoren der nachfolgenden Reformdebatte untersucht, um drittens Erklärungen für den Inhalt und die Ausgestaltung der schließlich durchgeführten Reform zu generieren. Die Analyse orientiert sich dabei an unterschiedlichen konzeptionellen Erklärungsfaktoren, wodurch auch ein Beitrag zur internationalen - bisher noch wenig ausdifferenzierten - politologischen Debatte über die Erklärung der Reformpfade von Mindestsicherungssystemen geliefert wird (vgl. dazu insb. Clegg 20I4; Jessoula et al. 20I4; Madama 2OII; Madama et al. 20I4; Natili 20I8). 
Methodisch handelt es sich um eine Einzelfallstudie, die den gegenständlichen Reformprozess mittels process tracing nachzeichnet und analysiert. ${ }^{2}$ Process tracing eignet sich zur Analyse von komplexen Phänomenen. Es werden dabei kausale Prozesse zwischen den unabhängigen Variablen bzw. erklärenden Faktoren und der abhängigen Variable, in unserem Fall die in den einzelnen Phasen dominante inhaltliche Entwicklung der Reformdebatte und das sich schließlich zeigende Reformergebnis, identifiziert. Explizit zielt process tracing auf die Identifizierung von theoretischen Erklärungen $\mathrm{ab}$, die über eine reine Prozessbeschreibung hinausgehen. Es wird der Hergang eines Prozesses rekonstruiert, um verallgemeinerbare Erklärungen zu finden bzw. zu überprüfen (vgl. George/Bennett 2005; Collier 20II).

Aus einer breiteren Perspektive ist eine Einzelfallstudie zu Österreich zum gegenständlichen Politikbereich aus dem Grund besonders interessant, dass es sich hier - wie oben bereits angeschnitten - aus international vergleichender Perspektive um einen abweichenden Fall handelt, weil im Gegensatz zu vielen anderen Ländern ursprünglich ein substantieller Ausbau sozialstaatlicher Leistungen für Outsider vorgesehen war bzw. diese Zielsetzung ursprünglich im Fokus der gegenständlichen Reformdebatte stand. Abweichende Fälle versprechen generell weiterführende Erkenntnisse, weil bei deren Analyse dargelegt werden kann, warum gerade für sie gängige Erklärungen nicht ausreichen. Damit eignen sich abweichende Fälle einerseits zur Hypothesengewinnung, andererseits können ebenso bestehende Hypothesen und konzeptionelle Überlegungen und Theorien verfeinert werden (vgl. Lijphart 1971; Mahoney 2007). Der Beitrag ist wie folgt strukturiert: Abschnitt 2 präsentiert konzeptionelle Überlegungen zur Erklärung der „Politik von Mindestsicherungsreformen“. Abschnitt 3 skizziert die erste Phase des gegenständlichen Reformprozesses, also das ursprüngliche Agendasetting und den Problemhintergrund. In Abschnitt 4 analysieren wir die inhaltlich bestimmende Phase des gegenständlichen Reformprozesses ab dem Jahr 2005 bis zur Beschlussfassung im Jahr 20I0, wo die Reform der Mindestsicherung in Österreich zu einem dominanten sozialpolitischen Projekt stilisiert wurde. In Abschnitt 5 erfolgt eine Diskussion und Zusammenfassung und der Beitrag schließt mit einem Ausblick, der auch kurz rezente Entwicklungen im gegenständlichen Politikfeld anspricht.

2 Für diese Studie wurden verschiedenste schriftliche Datenquellen verwendet. Systematisch wurden Partei- und Wahlprogramme betrachtet. Inhalte von Datenbanken über Parlamentsprotokolle, Pressemeldungen und Zeitungsberichte wurden nach den Stichwörtern, Sozialhilfe, Grundsicherung und Mindestsicherung durchsucht und analysiert, wobei der Schwerpunkt auf die Jahre 2005 bis 2010 gelegt wurde. Weiters wurden Berichte des Sozialministeriums und von Interessenorganisationen sowie Sekundärliteratur einbezogen.

\section{Ansätze zur Erklärung von Mindestsiche- rungsreformen}

Arbeiten zur Entwicklung von Mindestsicherungssystemen sind häufig deskriptiv und konzentrieren sich zumeist auf den Inhalt der jeweiligen Reformen bzw. ihre unmittelbaren - insbesondere verteilungspolitischen - Implikationen (Gough et al. 1997; Bahle et al. 20II; Lødemel/Moreira 20I4; Marchal/van Mechelen 2015; Wang/van Vliet 2016). Nur wenige Analysen liegen bisher international vor, die auf ein generelles Verständnis der politischen Logik von Reformprozessen und auf mögliche diesbezügliche erklärende Faktoren abzielen (vgl. insb. Clegg 20I4; Jessoula et al. 20I4; Madama 20II; Madama et al. 20I4; Natili 2OI4; 2OI8). Im folgenden Kapitel werden unterschiedliche theoretische Ansätze zur Erklärung von Mindestsicherungsreformen diskutiert. Zuerst werden der klassische funktionalistische Ansatz und der Machtressourcenansatz sowie die Erweiterung durch die Parteiendifferenzhypothese skizziert. Danach werden diese durch (neo-)institutionalistische Ansatzpunkte sowie durch Überlegungen über die Bedeutung von Ideen und "policy-entrepreneurs" erweitert. Dabei gehen wir grundsätzlich davon aus, dass einzelne Faktoren alleine, wie sie klassischen Theorieschulen entsprechen, den Fort- und Ausgang politischer Prozesse nur ungenügend erklären können. Und um einen bestimmenden Einfluss erkennen zu können, sind potentielle Erklärungsfaktoren zu einander in Bezug zu setzen (Guy Peters 20II).

Aus einer funktionalistischen Perspektive wäre grundsätzlich zu erwarten, dass ein wachsender Bedarf an sozialer Sicherung eine Expansion von Sozialleistungen nach sich zieht (Wilensky/Lebeaux 1958). Hinsichtlich der rezenten Nachfrage nach Mindestsicherungsleistungen kann jedoch argumentiert werden, dass hier ein kontingenter Effekt wirtschaftlicher Dynamik auf die Sozialpolitik (vgl. Siegel 2002, 44) einwirkt. Geringeres Wirtschaftswachstum, steigende Arbeitslosigkeit und eine Ausdifferenzierung von Chancen und Risiken auf den Arbeitsmärkten erhöhen auf der einen Seite die Nachfrage nach Mindestsicherungsleistungen. Auf der anderen Seite können aber wirtschaftliche Stagnation und ihre Rückwirkung auf öffentliche Haushalte kontraktive (also den Geldfluss mindernde oder bremsende) sozialpolitische Maßnahmen befördern.

Der soziale Problemdruck und die budgetären Verhältnisse stellen mit Sicherheit wichtige Bedingungen für den jeweiligen sozialpolitischen Entwicklungspfad dar. Für sich genommen erklären sie aber unzureichend, warum wann welche sozialpolitischen Entscheidungen getroffen werden, die eben auch von politischen Dynamiken und Institutionen abhängen (Flora/Alber 198I). $\mathrm{Zu}$ den bestimmenden Erklärungsfaktoren gehört aus Sicht der klassischen Parteiendifferenzhypothese die $\mathrm{Zu}$ - 
sammensetzung der jeweiligen Regierung (Hibbs 1977; Schmidt 1982), in der die Machtverteilung unterschiedlicher Klassen zum Ausdruck kommt.

Demnach forcieren vor allem sozialdemokratische Parteien eine sozialstaatliche Expansion, speziell wenn die Interessen der Arbeiterbewegung zugleich im außerparlamentarischen Bereich, sprich durch Gewerkschaften, stark vertreten sind (Korpi 1983). Ausgehend von der Debatte über die „New Politics of the Welfare State" (Pierson 1994; 200I) wurden solche Befunde ab den I990er Jahren - bezogen auf die nunmehrige Phase des „permanenter Austerität“ (Pierson 200I) - zunehmend in Zweifel gezogen. Die "politics of blame avoidence“ (d.h. der Schuldvermeidung) sorgen demnach dafür, dass ein breiter Rückbau von sozialpolitischen Leistungen von politischen Parteien aller Couleur vermieden wird, um sich Chancen auf eine Wiederwahl aufrecht zu erhalten. Zudem modifiziere das einmal etablierte Politikerbe die Präferenzen von Interessensorganisationen und WählerInnen und ebne durch unterschiedliche Parteien verfolgte sozialpolitische Strategien tendenziell ein.

Hinsichtlich der Politiken zur Mindestsicherung gibt es jedoch Argumente dafür, dass die Parteiendifferenzhypothese bzw. der Machtressourcenansatz nicht in ihrer klassischen Form zutreffen. Betreffend sozialdemokratische Parteien und Gewerkschaften kann argumentiert werden, dass sich deren strategische Position selbst in den Ländern, wo sie noch einen vergleichsweise großen Einfluss haben - vor dem Hintergrund sozioökonomischer Veränderungen grundlegend gewandelt hat. Insbesondere Rueda (2007; 2005) und King und Rueda (2008) haben eingewandt, dass sich die historische Klientel von sozialdemokratischen Parteien in nunmehrige "Insider" und „Outsider" aufspalte. Folglich stellt sich die Frage, welche dieser beiden Gruppen in erster Linie vertreten werden soll.

Maßnahmen, die den Interessen von Insidern entsprechen, sind solche, die deren arbeitsrechtliche und sozialpolitische Situation absichern und deren finanzielle Belastungen für andere Gruppen zumindest nicht erhöhen. Outsider bedürfen hingegen gut bzw. weiter ausgebauter sozialer Dienstleistungen und Mindestsicherungssysteme sowie qualitativ hochwertiger arbeitsmarktpolitischer Maßnahmen. Die Kosten dafür gingen aber zulasten der Insider. Sozialdemokratische Parteien und Gewerkschaften vertreten, so die These, tendenziell die Interessen von Insidern nachhaltiger als jene von Outsidern. Letzteres besonders dann, wenn die sogenannten Outsider, etwa die BezieherInnen von Mindestsicherung, eine noch vergleichsweise kleine und darum wahlstrategisch nicht besonders wichtige Gruppe darstellen (Bonoli 2007). Darüber hinaus setzt sich diese Gruppe überproportional aus jüngeren Personen, Frauen, Personen mit Migrationshintergrund oder gering Qualifizierten zusammen. Dies sind allesamt At- tribute, die mit einer vergleichsweise geringen eigenen politischen Organisationsfähigkeit in Verbindung gebracht werden (ibid.). Wichtig ist im gegenständlichen Zusammenhang darüber hinaus, dass diese Gruppen vielfach nicht zur traditionellen Stammklientel von Gewerkschaften gehören, die sich in ihrer Vertretung häufig auf vergleichsweise stabil beschäftigte "Normalarbeitnehmer" konzentrieren (vgl. Ebbinghaus 2006; für Österreich: Flecker/Herrmann 2009).

Eine Reihe internationaler Untersuchungen deuten in die Richtung, dass sozialstaatlicher Rückbau, der ab den I990er Jahren in praktisch allen hoch entwickelten Wohlfahrtsstaaten auf der Agenda stand, unter sozialdemokratischen Regierungen sogar stärker ausgefallen ist als unter anderen Regierungskonstellationen (vgl. vgl. Ross 2000; Green-Pedersen 2002). Die "politischen Kosten" (im Sinne nachfolgender Stimmverluste), so die These, fielen nämlich für sozialdemokratische Parteien geringer aus, weil diese nicht im Verdacht stünden, sozialpolitische Leistungen aus ideologischen Gründen zu reduzieren, sondern weil es aus budgetären Gründen oder Erhalt der Wettbewerbsfähigkeit "notwendig“ sei. Um einem drohenden Vertrauensverlust zu entkommen, können solche Maßnahmen zusätzlich auf wahlstrategisch wenig bedeutende Personengruppen beschränkt werden (Pierson 1994). Dies ist - neben anderem - Gegenstand der These einer „Dualisierung“ der Generosität sozialstaatlicher Leistungen, die eine Ausdifferenzierung von Chancen und Risiken auf den Arbeitsmärkten fortschreibt (Emmenegger et al. 2012). Outsider seien demnach nicht nur mit dem Problem konfrontiert, armutsfeste Erwerbseinkommen zu generieren. Darüber hinaus werde für diese Gruppe der Zugang zu Sozialtransfers zunehmend erschwert, an zusätzliche Konditionalitäten gebunden und/oder das Leistungsniveau gesenkt (Bothfeld/Betzelt 2013; Nelson 2013; Marchal et al. 20I4).

Die klassische Parteiendifferenzhypothese hingegen geht davon aus, dass in diese Richtung deutende politische Entscheidungen im Falle von (Mitte-)rechts-Regierungen noch drastischer ausfallen würden. Allerdings erscheint hier gleichfalls eine weitere Differenzierung allgemeiner Annahmen notwendig.

Generösen Sozialleistungen stehen liberale Rechtsparteien einerseits generell kritisch gegenüber. Zugleich ist aber durchaus zu erwarten, dass sie im Gesamtgefüge sozialstaatlicher Absicherung für eine größere Bedeutung von Mindestsicherungssystemen eintreten, allerdings verbunden mit einem Plädoyer für geringer ausgebaute andere sozialstaatliche Interventionen (vgl. Clegg 2013; Natili 20I4, 6; Jessoula et al. 20I4). Dabei wird ein Modell der residualen Sozialpolitik mit geringen sozialstaatlichen Ausgaben vertreten. Die Mindestsicherung ist in einem solchen Konzept für einen großen Teil der Arbeitslosen zuständig, wobei ein niedriges Leistungs- 
niveau und eine rigide Verpflichtung zu Erwerbs- und/ oder gemeinnütziger Arbeit die sozialen Rechte auf ein Minimum beschränkt.

Konservative christlich-soziale Parteien verfolgen gemäß der Parteiendifferenzhypothese vor allem eine starke soziale Absicherung des klassischen male-breadwinner-Modells durch Sozialversicherungsleistungen nach dem Äquivalenzprinzip, wobei sie zugleich die Bedeutung des Subsidiaritätsprinzips betonen (vgl. Huber/ Stephens 2000). Vor dem Hintergrund einer starken Einbindung von christlichen Gewerkschaften sind generöse Sozialversicherungslohnersatzleistungen und ein hoher Arbeitnehmerschutz für diese Parteien idealtypischer Weise häufig ein Anliegen. Des Weiteren stehen sie jedoch der Zentralisierung sozialer Leistungen grundsätzlich kritisch gegenüber (Kersbergen van/ Stahis 20IO). Noch verstärkt gilt dies nach einschlägigen Überlegungen für nationale Mindestsicherungssystemen mit eher generösen Zugangsregelungen, weil diese aus ihrer Sicht Praktiken und Werte familiärer und dezentraler gemeinschaftsbasierter sozialer Sicherung unterwandern könnten (vgl. Jessoula et al. 20I4).

Radikale Rechtsparteien forcieren besonders häufig eine Unterscheidung in "würdige“ (,deserving") und "unwürdige“ (,undeserving") soziale Gruppen. BezieherInnen von Mindestsicherungsleistungen (außer etwa alte oder kranke Personen) werden dabei oft dem Generalverdacht ausgesetzt, arbeitsunwillig zu sein. Es ist demnach wahrscheinlich, dass solche Parteien breit ausgebaute Mindestsicherungssysteme in besonderem Maß ablehnen werden (vgl. Natili 2014).

Für das faktisch sozialpolitische Agieren der Parteien ist zudem die Parteienkonstellation prägend. Konkret geht es dabei um die Frage, mit welchen anderen Kräften politische Parteien in einem Mehrparteiensystem um WählerInnenstimmen konkurrieren (vgl. Ferrera 1993; Häusermann et al. 20I0). Wenn sozialdemokratische Parteien mit der Konkurrenz anderer Linksparteien konfrontiert sind, werden sie eher auch die Interessen von sogenannten Outsidern auf die Agenda setzen (Anderson/Beramendi 2012). Wenn der Wettbewerb hingegen vor allem mit einer christlich-sozialen Partei stattfindet, dann treten sozialdemokratische Parteien beim Schutz von Insidern in Konkurrenz mit den ChristlichSozialen, und stellen die Interessen von Outsidern hintan: „The only viable avenue for social democratic parties is to moderate and effectively abandon their poor (,radi$\mathrm{cal}^{\prime}$ ) constituencies" (Iversen/Sodkice 2009, I2).

Für Positionen und Strategien von politischen Parteien, Interessensgruppen und anderen AkteurInnen ist auch die institutionelle Ausgestaltung des tradierten Systems sozialer Mindestsicherung wesentlich. (Neo-) institutionalistische Überlegungen verweisen einerseits auf das Phänomen der Pfadabhängigkeit und nehmen andererseits die Verteilung von politischen Kompetenzen und die sich daraus ergebenden Rahmenbedingungen in den Blick. Dabei spielt die Kompetenzverteilung zwischen dem Nationalstaat und der subnationalen Sphäre eine Rolle, da lokale und/oder regionalen AkteurInnen und Institutionen in der Mindestsicherung häufig Schlüsselfunktionen innehaben und somit mehr oder minder Vetopunkte bilden bzw. als Vetospieler agieren können (Pierson 1995). Inwiefern sich subnationale Kompetenzen in der Mindestsicherung auf einschlägige Reformprozesse auswirken, wurde bislang jedoch kaum in theoriegeleiteten und empirisch gesättigten Analysen untersucht. Bonoli/Champion (2015) gehen davon aus, dass subnationale Kompetenzen in Zeiten eines erhöhten Reformdrucks mannigfache Effekte zeigen können. Diese reichen von dezentraler Innovation über Versuche einer wechselseitigen Verschiebung von LeistungsbezieherInnen (Kosten und Verantwortung) zwischen unterschiedlichen Ebenen bis zu offen ausgetragenen Kompetenzkonflikten. Letzteres wird hier als Ausdruck eines innerstaatlichen Wettbewerbs interpretiert, um über Möglichkeiten zu verfügen, Problemlösungsfähigkeit zu signalisieren und damit politisches credit-claiming $\mathrm{zu}$ betreiben.

Betrachtet man diese verschiedenen Erklärungsansätze, so kann davon ausgegangen werden, dass im politischen Aushandlungsprozess immer ein Gemenge von Faktoren zum Tragen kommt. Gleichzeitig weisen einzelne Zugänge Schwachstellen auf, die wiederum einer Erweiterung durch andere Ansätze bzw. Überlegungen bedürfen. Gelten funktionalistische und machtressourcentheoretische Erklärungen vor allem für eine wohlfahrtsstaatliche Expansion, so begründen sich neo-institutionalistische oftmals in der Betrachtung eines etablierten sozialpolitischen Arrangements und von dessen Stabilität. Andere Überlegungen fokussieren auf Veränderungen und rücken den Wandeln in politischen Ideen und relevante Einflussnahmen von Entscheidungsträger/innen in den Vordergrund.

Solche Ansätze mittlerer Reichweite bringen neben "structure" auch vermehrt „agency" ins Spiel, um sozialpolitische Veränderungen erklären zu können (insb. Madama 20II). Nach unserer Lesart sind diese Überlegungen gegenüber "klassischen“ Ansätzen, wie etwa dem Funktionalismus, dem Parteiendifferenz- und Machtressourcenansatz oder dem (Neo-)Institutionalismus, anschlussfähig.

Deshalb berücksichtigen wir überdies die Rolle von individuellen AkteurInnen, so genannten "policy entrepreneurs", die politische Prozesse bei einem gegebenen "window of opportunity" beschleunigen oder in eine andere Richtung lenken können (Kingdon 1984). Zweitens behalten wir die Entwicklung inhaltlich dominanter „Ide$e n$ " im Auge. Interessen und Strategien von AkteurInnen können sich aus der Perspektive des diskursiven NeoInstitutionalismus vor dem Hintergrund gewandelter 
Interpretationsrahmen ändern. Und sich wandelnde Ideen können damit entscheidend $\mathrm{zu}$ institutioneller Transformation beitragen (Béland 2005; Schmidt 2008; Hay 2004).

Eine wesentliche Rolle kann dabei - so der gängige Befund - bei der Diffusion und Verfestigung bzw. unter Umständen selbst bei der Transformation von Ideen unterschiedlichen nationalen und insbesondere auch internationalen Akteursnetzwerken zukommen (Hall I993; Sabatier/Jenkins-Smith 1993). Zudem können sich verändernde dominante Ideen und "policy entrepreneurs" in Kombination zu ungewöhnlichen Koalitionen führen, die unter Umständen sogar eine Pfadabweichung ermöglichen. So genannte "coalition magnets" entstehen dann, wenn eine Idee, wie etwa soziale Ausgrenzung, von einem politischen Entrepreneur so forciert werden kann, dass ein soziales Problem in einem anderen Zusammenhang gesehen wird. Entscheidungsrelevant kann eine solche Konstellation dann werden, wenn sich wichtige Akteur/innen im politischen Prozess für die Idee begeistern, wobei diese bis dahin keinen gemeinsamen Handlungsbedarf gesehen haben (Béland/Cox 2016).

Im folgenden Abschnitt wird der politische Prozess vom Agendasetting bis zum Beschluss im Parlament dargestellt. Induktiv werden für jede Phase prägende Faktoren eruiert und erläutert, um im Diskussionsteil aus dem nachgezeichneten Prozess und mit Bezug auf die oben skizzierten Erklärungsansätze Hypothesen zu generieren.

\section{Agendasetting: Armutspolitik und/oder Arbeitsmarktpolitik?}

Grundsätzlich erfolgte die legistische Regelung und Implementierung der Sozialhilfe in Österreich in den I970er Jahren auf Ebene der Bundesländer. Davor hatte der Bund 1968 explizit auf die verfassungsrechtlich vorgesehene Grundsatzgesetzgebung verzichtet, nachdem mit den Bundesländern - trotz wiederholt ausformulierter Entwürfe - über eine solche keine Einigkeit erzielt werden konnte (Melinz 1996). Danach intensivierte sich die Debatte um eine Reform monetärer sozialer Mindestleistungen (in Form der damaligen Sozialhilfe) in Österreich auf breiter Basis erst wieder seit Mitte der I990er Jahre, was eine Reihe von Ursachen hat und auf Bestrebungen sehr unterschiedlicher AkteurInnen fußt.

$\mathrm{Zu}$ Beginn der I990er Jahre waren die Länder mit einer steigenden Nachfrage nach Leistungen der offenen Sozialhilfe ${ }^{33}$ konfrontiert, wobei dies für Wien schon früher zutraf (Köppl/Steiner 1989; Pfeil/Otter 2OII).

3 Damit sind Leistungen gemeint, die nicht im Rahmen der intramuralen Betreuung wie etwa Pflegeheimen gewährt werden.
Durch die Veränderungen in der Familien- und Beschäftigungsstruktur entstanden „neue soziale Risiken“, die von vorgelagerten Sicherungssystemen nur teilweise abgedeckt wurden. Oder es wurde durch gesetzliche Änderungen, insbesondere in der Arbeitslosenversicherung, sogar deren Risikoabdeckung reduziert (Obinger/ Tálos 20I0; Tálos/Mühlberger 1999). Die Daten zur Entwicklung der Zahl der Sozialhilfebezieherinnen während der ersten Hälfte der I99oer Jahre bilden diese erhöhte Nachfrage nicht ausreichend ab (Bock-Schappelwein 2004), vor allem weil der Zugang zur Sozialhilfe in dieser Zeit vielfach restriktiver gestaltet wurde (Dimmel 2008). Grundsätzlich standen die Länder einer Reform der offenen Sozialhilfe seit Mitte der I99oer Jahre offen gegenüber, da sie sich ähnlich wie bei der 1993 implementierten Pflegereform (Einführung des Pflegegeldes) unter anderem eine gewisse finanzielle Entlastung erhofften.

Die Initiative zu einer Reform der Sozialhilfe ging jedoch ursprünglich nicht von den Bundesländern aus. Bereits in der zweiten Hälfte der I980er Jahre fand eine öffentlichkeitswirksame Diskussion über ein „Grundeinkommen ohne Arbeit" statt, deren Ausgangspunkt das gleichnamige Buch (Wohlgenannt/Büchele 1985) der Katholischen Sozialakademie war. Die Thematik wurde in der Folge von zivilgesellschaftlichen AkteurInnen und zwei parlamentarischen Oppositionsparteien, den Grünen und dem Liberalen Forum (LIF), aufgegriffen. Die "Armutskonferenz" ${ }^{4}$ kurbelte die Debatte weiter an: Sie wurde 1995 als Netzwerk gegen Armut und soziale Ausgrenzung gegründet und band neben zivilgesellschaftlichen Organisationen WissenschafterInnen ein. Ihre zweite jährliche Tagung, die im Jänner 1997 in Salzburg stattfand, wurde unter das Thema „Soziale Grundsicherung" gestellt. Sie zeigte in dem Sinn einen dauerhaften Effekt, dass der bedarfsgerechte Ausbau sozialer Mindestleistungen im Anschluss daran bei zentralen zivilgesellschaftlichen Organisationen in Österreich zu einer - bzw. vielfach sogar der - zentralen armutspolitischen Forderung avancierte.

Wie schon oben erwähnt, wurde die Reformdiskussion zuerst von zwei Oppositionsparteien in den parlamentarischen Raum eingeführt. Die Grünen stellten I995 ein Pensionsmodell vor, das eine Grundsicherung unabhängig von Arbeit und Familienstand enthielt. Im Oktober 1998 folgte ein umfassendes Konzept einer "Grünen Grundsicherung", das ein generöseres Leistungsniveau, umfassende Möglichkeiten der Karenzierung und universelle Leistungen für Kinder vorsah (Die Grünen 1998). Das LIF (1997) hatte bereits 1997 das Modell einer „Bedingungslosen Grundsicherung“ präsentiert, und zwar in Form einer Negativsteuer von monat-

4 In der Armutskonferenz sind mehr als 30 soziale Organisationen sowie Bildungs- und Forschungseinrichtungen aktiv. 
lich ca. 750 EUR. Dieses Modell sah, anders als jenes der Grünen, von der Bereitschaft zur Erwerbstätigkeit ab. Andere Sozialleistungen, etwa Arbeitslosenversicherung, sollten jedoch gestrichen werden.

Mit explizitem Bezug auf die vorangegangene Veranstaltung der Armutskonferenz brachte das LIF am 29. Jänner 1997 im Parlament einen Entschließungsantrag „betreffend Schaffung eines Bundessozialhilfegesetzes“ ein, ${ }^{5}$ da ein „bundeseinheitliches Sozialhilfegesetz [...] eine wichtige und dringende Voraussetzung für die Sicherung sozialer Grundrechte jedes Menschen in diesem Land“ sei. Es sollte Richtsätze für Einzelpersonen und Haushaltsgemeinschaften vereinheitlichen, Leistungen mit Rechtsanspruch und klar ausgestalteten Bedingungen (Erfordernis und Zumutbarkeit des Einsatzes der eigenen Arbeitskraft) festhalten, Schonvermögen bestimmen und den Regress beschränken. Die Verhandlungen wurden im Ausschuss für Arbeit und Soziales weitergeführt. Seitens der ÖVP und der SPÖ wurde ein gemeinsamer Abänderungsantrag eingebracht und von diesen beschlossen. ${ }^{6}$ Neben der ÖVP und der SPÖ stimmte das LIF dem abgeänderten Entschließungsantrag im Nationalrat zu, nicht jedoch die Grünen, weil sie diesen Antrag als inhaltlich zu schwach empfanden. Auch die FPÖ (Freiheitliche Partei Österreich), von der in der gegenständlichen Nationalratsdebatte keine Wortmeldung erging, stimmte nicht zu. ${ }^{7}$ Inhaltlich weniger weitreichend als der Vorstoß des LIF wurde in der Entschließung "(d)ie Bundesregierung, insbesondere die Bundesministerin für Arbeit, Gesundheit und Soziales, [...] ersucht, mit den Ländern Gespräche über die Weiterentwicklung der Sozialhilfe aufzunehmen“.

Dem parlamentarischen Auftrag nachkommend wurde 1998 beim Sozialministerium eine mehr als 40-köpfige ExpertInnenarbeitsgruppe (ohne Beteiligung der Länder) eingerichtet, die später den Bericht „Einbinden statt Ausgrenzen“ vorlegte (Haberbauer et al. 1999). Explizit wird im Vorwort hervorgehoben, dass die dargelegten Reformoptionen die Sichtweisen der ExpertInnen wiedergeben, und nicht mit Stellungnahmen von Organisationen gleichzusetzen sind (neben wissenschaftlichen Einrichtungen etwa von Sozialministerium, Arbeiterkammer, Gewerkschaft oder Arbeitsmarktverwaltung). Der Bericht der Arbeitsgruppe kam zum Schluss, dass Leistungen, die auf sozialversicherungsrechtlichen oder universalistischen Prinzipien basieren, ausgebaut werden sollten, um die Bedeutung bedarfsgeprüfter Leistungen hintanzuhalten. Die

5 Vgl. 388/A XX.GP; http://www.parlament.gv.at/PAKT/VHG/XX/ A/A_oo388/index.shtml.

6 Vgl. Vgl. 880 der Beilagen XX. GP; http://www.parlament.gv.at/ PAKT/VHG/XX/I/I_oo880/fname_I39756.pdf.

7 Vgl. Stenographisches Protokoll, 88. Sitzung des Nationalrates der Republik Österreich, XX. Gesetzgebungsperiode, Mittwoch, 8. Oktober 1997, Internet: http://www.parlament.gv.at/PAKT/VHG/ XX/NRSITZ/NRSITZ_ooo88/fname_II4I65.pdf.
Ausgleichszulage der Pensionsversicherung sollte als einheitlicher Referenzwert für bedarfsgeprüfte Unterstützungen herangezogen werden. Zugleich sollten einkommensgeprüfte Leistungen (wie Notstandshilfe, Ausgleichszulage, Zuschuss zum Karenzgeld) armutsfester gestaltet werden. Weiters wurde empfohlen die Sozialhilfe durch eine „Bedarfsorientierte Mindestsicherung“ (BMS) zu ersetzen. Diese sollte weiterhin residual zu anderen Sozialleistungen bleiben, aber wesentlich leichter zugänglich sein, bundesweit vereinheitlicht werden und eine höhere Rechtssicherheit garantieren sowie eine Betreuungskomponente durch soziale Dienste und eine Absicherung in der Kranken- und Pensionsversicherung beinhalten. Wichtig ist, dass im Bericht der ExpertInnenarbeitsgruppe ein armutspolitischer gegenüber einem arbeitsmarktpolitischen Standpunkt überwiegt: „Armut wurde nicht nur als Ergebnis fehlender/eingeschränkter Erwerbsarbeit angesehen, sondern als multidimensionaler Prozess, dem ebenso zu begegnen sei“ (Dimmel 2008, 31). Ohne dass die Ergebnisse dieser Arbeitsgruppe vorerst breiter aufgegriffen worden wären, wurde 1999 mit dem Ziel einer Vereinheitlichung der Qualitätsstandards in der Sozialhilfe eine weitere Arbeitsgruppe bestehend aus den LandessozialhilfereferentInnen unter Leitung des Sozialministeriums installiert (Pfeil/Otter 20II).

Inzwischen hatte sich auf Bundesebene die Regierungskonstellation geändert. Statt mit der SPÖ regierte die ÖVP ab dem 4. Februar 2000 in einer Koalition mit der FPÖ. Im Regierungsprogramm wurde angekündigt, dass die Leistungen von Bund und Ländern hinsichtlich der Existenzsicherung besser aufeinander abgestimmt werden sollten und für BezieherInnen an einer Stelle zugänglich sein sollten. Ein One-Desk-System sollte für die Vermittlung in den Arbeitsmarkt und die Auszahlung von Leistungen beim Arbeitsmarktservice (AMS) eingeführt werden. Sozialhilfe- und NotstandshilfebezieherInnen sollten dazu verpflichtet werden, „Gemeinwesensarbeit“ zu verrichten, und dafür ein „Bürgergeld“ in Form eines bis zu 20-prozentigen Aufschlages erhalten (Bundeskanzleramt 2000). Nach den vorgezogenen Nationalratswahlen vom November 2002 wurde erneut eine Koalitionsregierung aus ÖVP und FPÖ gebildet. Das neue Regierungsprogramm sah nunmehr vor, die Notstandshilfe in eine per Vereinbarung gemäß Art. 15a B-VG ${ }^{8}$ oder per Sozialhilfegrundsatzgesetz harmonisierte „Sozialhilfe neu“ überzuführen, die weiterhin bei den Ländern angesiedelt bleiben sollte. Parallel sollte

8 Bei Vereinbarungen gemäß Artikel I5a der Bundesverfassung (B-VG) handelt es sich dabei um einen innerstaatlichen Vertrag zwischen dem Bund und den Ländern, die dabei über Angelegenheiten ihres jeweiligen Wirkungsbereichs Übereinkünfte treffen, die verbindlich sind. Solche Vereinbarungen sind jedoch in der Regel nicht mit unmittelbaren Sanktionierungsmöglichkeiten ausgestattet, die im Fall eines Zuwiderhandelns wirksam werden würden. 
ein kollektivvertraglicher Mindestlohn von I.ooo Euro im Monat etabliert werden (Bundeskanzleramt 2003).

Die Länder wollten weitergehenden Veränderungen nurunter derVoraussetzung zustimmen, dass der Bund in seinem Verantwortungsbereich, insbesondere Arbeitslosenversicherung, Leistungsverbesserungen durchführt (Pfeil/Otter 2OII), was aber im Regierungsprogramm nicht vorgesehen war. Dessen ungeachtet erarbeitete die Arbeitsgruppe „Weiterentwicklung des Sozialhilferechts" der LandessozialhilfereferentInnen in Kooperation mit dem Experten Walter J. Pfeil einen Entwurf für eine Art. 15a-B-VG-Vereinbarung zwischen Bund und den Ländern, die im März 2004 im Rahmen einer Enquete der Volksanwaltschaft vorgestellt wurde. Wesentliche Eckpunkte waren die Anhebung der Sozialhilferichtsätze auf das Niveau der Ausgleichszulage, die Etablierung eines One-Desk-Prinzips beim AMS, die Integration nicht versicherter SozialhilfebezieherInnen in die reguläre Krankenversicherung und eine Erhöhung von Leistungen der Arbeitslosenversicherung. Im Juni 2004 forderte die LandessozialreferentInnenkonferenz den Bund auf, seine grundsätzliche Position vorzulegen. ${ }^{9}$ Seitens des Bundesministeriums für Soziale Sicherheit und Generationen und des Bundesministeriums für Wirtschaft und Arbeit wurde keine offizielle Stellungnahme abgegeben. Der von den Ländern monierte Verbesserungsbedarf im Bereich der Ausgleichszulagenrichtsätze, des Arbeitslosengeldes sowie der Notstandshilfe war dort offensichtlich kein Thema (vgl. Dimmel 2008, 32). Nur das Bundesministerium für Gesundheit und Frauen (BMGF) errechnete die finanziellen Mehrbelastungen durch eine Einbeziehung nicht versicherter SozialhilfebezieherInnen in die Krankenversicherung (Pfeil/Otter 20II).

Diese Entwicklungen indizieren eine weitgehende Politikblockade zwischen Bund und Ländern. Der Bundesregierung waren weder die Verbesserung bei Mindestsicherungsleistungen noch der Leistungsausbau in den vorgelagerten Sozialversicherungsleistungen ein Anliegen. Vielmehr sollte die „Beschäftigungswilligkeit" von LeistungsbezieherInnen im Rahmen einer ausreichenden Anreizkompatibilität von Sozialleistungen sichergestellt werden (vgl. Fink 2006). Umgekehrt sahen sich die Länder mit einer Sozialhilfereform ohne Einbindung der Betroffenen in das AMS-System überfordert, zumal sie keine zusätzlichen eigenen Budgetmittel aufwenden wollten (Tálos 2008a). Zugleich war für den Bund die ursprünglich geplante Überführung der Notstandshilfe in die Sozialhilfe wegen eines diesbezüglichen Widerstandes der Länder keine durchsetzbare Option.

9 Inhaltlich sollte der Bund dabei nach den Vorstellungen der Länder vor allem auf Fragen finanzieller Mindeststandards, auf die Weiterentwicklung der Notstandshilfe und die Krankenversicherung für SozialhilfeempfängerInnen eingehen.

\section{Die Mindestsicherung als zentrales sozial- politisches Projekt und dessen schrittwei- ser inhaltlicher Wandel}

Damit existierte zwischen dem Bund und den Ländern betreffend eine weitreichende Reform der Sozialhilfe eine Art Pattsituation. Zugleich standen die SPÖ wie auch die Gewerkschaften einer umfassenderen Reform der Sozialhilfe lange ursprünglich skeptisch gegenüber (Tálos 2008a, I60; 2003, I69ff.). Soziale Risiken sollten aus Sicht der Sozialdemokratie in erster Linie durch das Sozialversicherungssystem bearbeitet werden. Im Zuge der parteiinternen Vorbereitungsphase zum Nationalratswahlkampf 2006 änderten sich jedoch die diesbezüglichen Signale, als zur Schärfung des inhaltlichen Profils der SPÖ ab Anfang 2005 von politikfeldspezifischen "Kompetenzteams“ Maßnahmenvorschläge erarbeitet wurden. Das Kernteam im Sozialbereich bildeten zwei damalige Sozial-LandesrätInnen, nämlich Gabriele Schaunig aus Kärnten und Erwin Buchinger aus Salzburg, sowie Richard Leutner (Österreichischer Gewerkschaftsbund, ÖGB) und Heidrun Silhavy (Sozialsprecherin im Nationalrat). Beim ersten öffentlichkeitswirksamen Auftritt des Kompetenzteams wurde im Jänner 2004 unter anderem gefordert, die Sozialhilfe durch eine neue „bedarfsorientierte Grundsicherung" zu ersetzen. ${ }^{10}$ Im Mai 2005 lautete eines von zehn präsentierten Zielen des Kompetenzteams, die „Armut in Österreich [zu] beseitigen (bedarfsorientierte Mindestsicherung) ${ }^{{ }{ }_{\mathrm{II}}}$.

Seitens der SPÖ wurde das Thema jedoch vorerst nicht weiter öffentlich forciert. Weit präsenter waren diesbezüglich die Grünen, die im Wahlkampf für die Wiener Landtags- und Gemeinderatswahl vom Oktober 2005 die Forderung nach einer neuen Mindestsicherung als eines ihrer Kernthemen präsentierten. ${ }^{12}$ Die SozialdemokratInnen brachten ihr Konzept einer „bedarfsorientierten Grundsicherung" erst in der Hochphase des nächsten Nationalratswahlkampfes ab dem Spätsommer 2006 in diesen ein. Allerdings gab es kaum öffentliche Stellungnahmen seitens der SPÖ dazu, ${ }^{13}$ wohingegen die Grünen abermalig ihr Modell der Grundsicherung als maßgebliche sozialpolitische Forderung platzierten. ${ }^{14}$ Insgesamt verdichtet sich vor diesem Hintergrund

IO APA Presseaussendung, 2APA708 II I2.01.2005, „SP-Konferenz: Sozialteam stellt Ziele vor“.

II OTS Presseaussendung, OTSi67 II 02.05.2005., „Kompetenzteam Soziales: SPÖ will Modernisierung und Stärkung des Sozialstaats“.

I2 Das dort im Anschluss getroffene Arbeitsübereinkommen zwischen SPÖ und den Grünen über eine „projektbezogene Zusammenarbeit" beinhaltet keine Reform der Wiener Sozialhilfe in Richtung einer neuen Grund- oder Mindestsicherung, wobei seitens der SPÖ argumentiert wurde, dass ein solcher Schritt einer „bundespolitischen Mitarbeit“ bedürfe (vgl. OTSIO4 II I3.OI.2006).

I3 Vgl. z.B. OTS Presseaussendung, OTS29I II 29.09.2006: „SPÖWahlkampfabschluss: Ein Kanzler, der Wort hält, statt eines Kanzlers der gebrochenen Versprechen".

I4 Vgl. APA Presseaussendung, APA623 II I8.09.2006, „NR-Wahl: Grüne starten Intensivwahlkampf“. 
der Eindruck, dass die angestrebte Reform der Sozialhilfe innerhalb der SPÖ kein von einer breiten Basis getragenes Kernthema war. Die nur punktuelle öffentliche Hervorhebung deutet darüber hinaus darauf hin, dass wohl einige Zweifel über die Wahlkampftauglichkeit des Themas existierten.

Als stärkste Kraft aus den Wahlen am I. Oktober hervorgehend startete die SPÖ in Folge Koalitionsverhandlungen mit der ÖVP. Sozialpolitische Themen wurden bei der SPÖ von der damaligen Salzburger Landeshauptfrau Gabi Burgstaller, dem Gewerkschafter Friedrich Haberzettel und von Erwin Buchinger verhandelt. ${ }^{15}$ Letzterer hatte zuvor beim Entwurf des SPÖ-Modells einer bedarfsorientierten Grundsicherung mitgearbeitet (vgl. Buchinger 2006). Erst zu diesem Zeitpunkt wurde die Reform der Sozialhilfe in Form einer Mindestsicherung zum Gegenstand einer intensiven und breit geführten öffentlichen politischen Debatte. ${ }^{16}$ Die SPÖ betonte dabei, dass das von ihr forcierte Modell kein bedingungsloses Grundeinkommen darstelle und im Wesentlichen auf einer Vereinheitlichung der Sozialhilfe basiere. Am 20.12.2006 wurde schließlich öffentlich, dass ÖVP und SPÖ eine Übereinkunft erzielt hatten, deren Umsetzung mit einer Vereinbarung gemäß Art. I5a B-VG zwischen dem Bund und den Ländern von Anfang an erst für das Jahr 2010 angepeilt war. ${ }^{17}$ Die Konzessionen der SPÖ in den Verhandlungen mit der ÖVP waren einerseits die Zustimmung zum sogenannten Flexicurity-Paket $2007^{18}$, die weitreichende Verschlechterungen für Arbeitslose hinsichtlich von Wegzeiten und betreffend der Verfügbarkeit und Teilnahme an gemeinnützigen Beschäftigungsprojekten umfasste, sowie andererseits die anhaltende Zuordnung der Arbeitsmarktagenden zu einem Wirtschafts- und Arbeitsministerium unter Leitung der ÖVP.

Das Regierungsprogramm 2007 benannte „eine weitere Verstärkung der Armutsbekämpfung zur Senkung der Zahl der Armutsgefährdeten und akut Armen" (Bundeskanzleramt 2007, I09) als explizites Ziel, wobei ausgeführt wurde, dass „das Instrument dafür [...] die Einführung einer bedarfsorientierten Mindestsicherung sein [soll]" (ibid.).

I5 Vgl. APA Presseaussendung, APA756 II 19.10.2006, „Koalition: Gruppenphase eröffnet - Soziales tagte ergebnislos".

I6 Vgl. z.B.: APA Presseaussendungen: APA093 II I9.II.2006, "Grundsicherung für die IV ,nicht vorstellbar'“; APA334 II 24.IO.2006, „Koalition: Grüne pochen auf Grundsicherung. Utl.: Öllinger: ,SPÖ bei Verhandlungen unverbindlich“ - ,SPÖ-Modell mangelhaft'“; AP428 II 22.10.2006, Grundsicherung - Bischof Schwarz: SPÖ-Konzept guter Ansatz; APA326 II 22.II.2006, „Koalition: VP-Leitl kann sich Grundsicherung vorstellen“; APA352 II 24.II.2006, „Koalition: Bartenstein legt Gegenmodell zur SPÖGrundsicherung vor".

17 Vgl. APA Presseaussendung APAO44 II 21.12.2006, „Koalition: Grundsicherung wird Thema beim Finanzausgleich; Utl.: Länder wollen Beitrag des Bundes - Verhandlungen über einheitliche Sozialhilfe“.

I8 Vgl. https://www.parlament.gv.at/PAKT/VHG/XXIII/I/I_Oo298/ index.shtml
Die monatliche Höhe der Mindestsicherung sollte, bei I4 jährlichen Auszahlungen, dem Ausgleichszulagenrichtsatz der Pensionsversicherung entsprechen, was als neue administrative "Armutsgefährdungsgrenze“ dargelegt wurde. Zur Implementierung der Mindestsicherung wurde vereinbart, in einem ersten Schritt den Ausgleichszulagenrichtsatz anzuheben. Überdies sollte ein Mindestlohn auf Basis eines Generalkollektivvertrags in Höhe von Iooo Euro sichergestellt werden. Als weitere flankierende Maßnahme wurde „unter dem Vorbehalt der Umsetzung einer bedarfsorientierten Mindestsicherung in den Bundesländern" (ibid., IIO) im Regierungsprogramm vorgesehen, dass die Notstandshilfe der Arbeitslosenversicherung bei niedrigen Leistungen angehoben wird. ${ }^{19}$ Zugleich sollten in der Notstandshilfe Partnereinkommen nur dann berücksichtigt werden, wenn dies nicht zu einem Haushaltseinkommen unter dem Familienausgleichszulagenrichtsatz (zuzüglich Kinderzuschläge) führt. Die Verbesserungen in der Notstandshilfe verfolgten dabei auch das Ziel, die Nachfrage nach Leistungen aus der Sozialhilfe bzw. der Mindestsicherung zu reduzieren.

Für arbeitsfähige Personen sollte Arbeitswilligkeit weiterhin eine Voraussetzung zum Leistungsbezug bleiben, im Falle von Langzeitarbeitslosigkeit verbunden mit einer Verpflichtung zu Weiterbildungsmaßnahmen und mit der Einbindung in "gemeinnützige Arbeitsprojekte“. Die Einkommens- und Vermögensüberprüfung sollte weiterhin durch die Sozialhilfeträger stattfinden. Die Antragsverwaltung, die Leistungsauszahlung und die Betreuung zur Reintegration in den Arbeitsmarkt sollten hingegen für arbeitsfähige BezieherInnen - „mit dem Ziel der Erreichung eines One-Stop-Shops" (ibid., III) - durch das AMS getätigt werden. Das Regierungsprogramm sah vor, dass Vermögen mit wenigen Ausnahmen weiterhin verwertet werden müssen. Zur Frage der Krankenversicherung finden sich im Regierungsprogramm keine Hinweise, obwohl dies Teil der Regierungsverhandlungen gewesen war. ${ }^{20}$

Die Ausführungen im Regierungsprogramm entsprechen damit in weiten Teilen dem zuvor durch die SPÖ vorgestellten Mindestsicherungskonzept (vgl. Buchinger 2006). Von den Grünen und von einer Reihe sozialer NGOs wurde kritisiert, dass diese Reform für sich genommen nicht weit genug gehe, um Armut und Armutsgefährdung nachhaltig und substanziell zu

I9 Erhöhte Nettoersatzrate des Arbeitslosengeldes von bis zu 60 Prozent bzw. im Fall von Familienzuschlägen von bis zu 80 Prozent als Bezugsgröße zur Berechnung der Notstandshilfe.

20 Vgl. APA Presseaussendung: APA37O II O4.I2.2006, „Landeshauptleute: Ja, aber ... zur Grundsicherung. Utl.: Verhandlungen über finanzielle Aufteilung gefordert“. 
reduzieren, ${ }^{21}$ und dass die Gefahr bestehe, dass die Mindestsicherung nur eine unzureichend adaptierte Version der alten Sozialhilfe werde. ${ }^{22}$

Jene Teile des Paketes, die nicht unmittelbar die Sozialhilfe betrafen, konnten relativ rasch umgesetzt werden. Noch bevor es zwischen SPÖ und ÖVP zu einem Regierungsübereinkommen kam, wurde einstimmig der Ausgleichszulagenrichtsatz für das Jahr 2007 auf 726 Euro angehoben. ${ }^{23}$ Mitte 2007 beschlossen die Sozialpartner in einer Grundsatzvereinbarung zwischen ÖGB und Wirtschaftskammer, die Branchenkollektivverträge für die niedrigsten Lohngruppen bis 2009 auf mindestens Iooo Euro brutto anzuheben. ${ }^{24}$ Im Rahmen des so genannten „Flexicurity-Paketes" ${ }^{{ }^{25}}$ vom Dezember 2007 wurden so genannte „Freie DienstnehmerInnen“ in die Arbeitslosenversicherung einbezogen und für Selbstständige wurde eine freiwillige Versicherung in der Arbeitslosenversicherung ermöglicht (BMSK 2009). Im Gegenzug wurden (s.o.) unter anderem die Zumutbarkeitsbestimmungen in der Arbeitslosenversicherung verschärft. Zwar forderten ArbeitnehmerInnenvertreterInnen zugleich eine sofortige Erhöhung der Nettoersatzrate in der Arbeitslosenversicherung (Tálos 2008b), aber die Regierung argumentierte, dass dies ein Teil der Mindestsicherungsvereinbarung mit den Ländern sein müsse.

Die unmittelbare Reform der Sozialhilfe und damit die Einführung der bedarfsorientierten Mindestsicherung wurde dagegen nicht rasch umgesetzt, obwohl bereits Anfang 2007 eine diesbezügliche Arbeitsgruppe aus VertreterInnen des Bundes, der Länder, der Städte und Gemeinden sowie der Sozialpartner eingerichtet worden war. Anders als unter der Vorgängerregierung von ÖVP und FPÖ war dabei die Zusammenlegung von Sozial- und Notstandshilfe kein Thema, unter anderem weil die vorangegangenen und in diese Richtung deutenden Reformen in Deutschland jedenfalls für manche Beobachter ihren Glanz schon verloren hatten. So meinte der damalige Sozialminister Buchinger $(2008,8)$, dass „Fehler [zu] vermeiden [seien], die in anderen Ländern gemacht

2I Vgl. z.B. die folgenden APA- und OTS-Presseaussendungen: APA263 II 26.02.2007, „Grüne über Buchingers Erbe in Salzburg: Viel Schein, wenig Sein“; OTS 022 II IO.OI.2007, „Attac: Große Koalition setzt neoliberalen Kurs fort“; APA 240 II 05.0I.2007, „Koalition - Grüne warnen vor ,Koalition der Lippenbekenntnisse "“.

22 Vgl. z.B. APA Presseaussendung: APA272 II 22.12.2006, „Koalition: Diakonie sieht in Mindestsicherung kaum Verbesserungen. Chalupka: Ohne Glitzerpapier ist Mindersicherung nur alte Sozialhilfe".

23 Vgl. APA Presseaussendung: APA223 II 15.12.2006, „Nationalrat: Mindestpension angehoben - Rot-Schwarzer Knatsch".

24 Dies betraf aber nur noch wenige Beschäftigte, da die Anzahl der Vollzeiterwerbstätigen, die weniger verdienten, schon zuvor von $243.000 \mathrm{im} \mathrm{Jahr} 2003$ auf $30.000 \mathrm{im} \mathrm{Jahr} 2007$ zurückgegangen war (vgl. Hermann 2009).

25 Vgl. APA-Presseaussendung: APAo7II II, WI 04.I2.2007, „Nationalrat: Schärfere Zumutbarkeitsbestimmungen für Arbeitslose Utl.: Freie Dienstnehmer bekommen künftig Arbeitslosengeld“. wurden, z.B. in Deutschland mit dem ,Hartz IV'-Modell“. Über die Leistungshöhe der Mindestsicherung, definiert als Mindeststandard (und nicht wie davor als Richtsatz, der im Einzelfall unter- und überschritten werden konnte), I4 jährliche Zahlungen, einen höheren Leistungssatz für Alleinerziehende, Ausnahmen für den Einkommenseinsatz sowie Regelungen zum Einsatz der Arbeitskraft konnte zügig Übereinstimmung hergestellt werden (Pfeil/Otter 2OII). Zugleich verzichteten die Länder und Gemeinden weder auf die Möglichkeit der Reduktion der Mindestsicherung bei niedrigeren Wohnkosten noch auf eine grundbücherliche Sicherstellung der Unterstützungsleistung. Der sogenannte Regress wurde dahingehend reformiert, dass BezieherInnen selbst die vorherige Mindestsicherungsleistung im Fall späterer Einkünfte nicht mehr zurückzahlen müssen. Unterhaltspflichten für nahe Angehörige nach dem Allgemeinen Bürgerlichen Gesetzbuch blieben aber bestehen. Dadurch wurde die Zielsetzung, dass die Mindestsicherung einem zeitgemäßen Verständnis von Unterhaltsverpflichtung entsprechen soll (Pfeil 2008), nur teilweise erfüllt.

Zugleich wurde der "One-Stop-Shop“ für arbeitsfähige LeistungsbezieherInnen sukzessive verwässert. Das AMS hätte dabei nach den ursprünglichen Plänen die Entgegennahme von Anträgen, deren Überprüfung sowie die Berechnung und die Überweisung der Leistung getätigt. Die Bestimmung zur Einkommens- und Vermögensüberprüfung wäre überdies stark an die der Notstandshilfe oder der Ausgleichszulage angelehnt worden (Pfeil/Otter 20II). Die Länder wehrten sich jedoch gegen den Verlust der Kontroll- und Weisungsrechte ohne eine gleichzeitige Kostenübernahme durch den Bund. Die Bundesländer Vorarlberg und Niederösterreich stimmten in einer ersten internen Abstimmungsrunde gegen eine umfassende Übertragung der Kompetenzen für die Zahlung der Mindestsicherungsleistung an das AMS. ${ }^{26}$ In beiden Bundesländern wurden neben den Landeshauptmännern auch die Soziallandesrätinnen durch die ÖVP gestellt. Man einigte sich schließlich darauf, dass das AMS nur mehr vorüberprüfen solle. ${ }^{27}$

Mit April 2008 wurde ein Ministerialentwurf über eine Art.-I5a-B-VG-Vereinbarung zwischen Bund und Ländern zur Stellungnahme ausgesandt (vgl. I88/ME, XXIII Gesetzgebungsperiode). Das AMS wandte ein, dass es hoheitsrechtliche Aufgaben von einer Landesbehörde übernehmen würde, wenn es Vorüberprüfungen durchführen sollte (19/SN-I88/ME, XXIII. Gesetzgebungsperiode). In Folge wurde die Rolle des AMS dahingehend modifiziert, dass es in Sachen Mindestsicherung als reine Informations- und Antragsstelle fungiert und die

\footnotetext{
26 Vlg. Der Standard, 27. 2. 2008, „Warten auf Mindestsicherung“; Der Standard, 3. 4. 2008, „Länder machen Weg frei für Mindestsicherung".

27 Vgl. Der Standard, 9. I. 2008, „Unsichere Mindestsicherung“; Der Standard, II. 4. 2008, „Bund und Länder erzielten Einigung“.
} 
Anträge "ungeprüft" an die Mindestsicherungsbehörde weiterleitet, damit es nicht als Bundesorganisation in der mittelbaren Landesverwaltung tätig werden muss. ${ }^{28}$

Die Finanzausgleichsverhandlungen zwischen Bund und Ländern brachten einen weiteren Erfolg für Länder und Gemeinden, da die mit der Mindestsicherung für die Länder einhergehenden Zusatzkosten mit EUR 50 Mio. pro Jahr begrenzt wurden. Würden höhere Aufwände anfallen, wären weitere Verhandlungen notwendig (Art. 2I der Art.-I5a-B-VG-Vereinbarung). Bei der Krankenversicherung und bei den Mitteln für die Arbeitsmarktpolitik hatten die Bundesländer keine Einwände, da die Mehrkosten beim Bund anfielen.

Die weiteren Entwicklungen brachten auch die von Beginn an vorgesehenen I4 Zahlungen pro Jahr zu Fall. Zuerst endete die bestehende Koalitionsregierung vorzeitig und der Nationalrat beschloss im Juli Neuwahlen für Ende September 2008 (8I4/A, XXIII. Gesetzgebungsperiode). Die Gesetzesvorlage zur Art.-I5a-B-VG-Vereinbarung war bereits zuvor im Ministerrat akkordiert worden ${ }^{29}$ und mit Anfang August hatten acht Länder der Vereinbarung formal zugestimmt. Allerdings verzögerte das Land Kärnten in der Person von Jörg Haider den weiteren Prozess und argumentierte, dass es sein eigenes Mindestsicherungsmodell beibehalten wolle, das mehr Arbeitsanreize und höhere Leistungen für Familien garantiere. ${ }^{30}$

Im anschließenden Wahlkampf dominierten Fragen der Reform der Sozialhilfe nicht. Zwar kritisierten die SPÖ und NGOs, dass Kärnten bzw. das BZÖ (Bündnis Zukunft Österreich) ${ }^{31}$ eine bundesweite Einigung über die Mindestsicherung verhindert hätten..$^{32}$ Im Gegensatz zu vorangegangenen Wahlkämpfen haben die Grünen und das LIF das Thema einer Grund- bzw. Mindestsicherung

28 Vgl. Der Standard, IO. 4. 2008, „Mindestsicherung: Start 2009 wird knapp“.

29 Vgl. Der Standard, 5. 6. 2008, „Koalition unter Druck“.

30 Vgl. Der Standard, 7. 8. 2008, „Buchinger ,Habe für Grundposition des Offenen Briefes Sympathie'“; Der Standard, 27. 8. 2008, "Annäherung bei Mindestsicherung"; Die Presse, 13.9.2008, „Sozialpolitik: Mindestsicherung kommt nicht mehr vor den Wahlen".

3I Das Bündnis Zukunft Österreich (BZÖ) wurde im April 2005 von Mitgliedern der Freiheitlichen Partei Österreichs (FPÖ) um Jörg Haider gegründet. Die gegenständlichen Verhandlungen fallen in die Zeit der Kärntner Landesregierung „Haider III“. Aufgrund des Proporzsystems waren an der Landesregierung je drei Vertreter von FPÖ (ab 2005 BZÖ) und SPÖ sowie ein Vertreter der ÖVP beteiligt.

32 Vgl. z.B. die folgenden APA- und OTS-Presseaussendungen: OTSo05I II II.08.2008, „Caritas-Präsident bestürzt über Scheitern bei Mindestsicherung, Utl: Küberl: ,Arme kommen im Wahlkampf unter die Räder - Bund und Länder sollen geplante Regelungen einzeln umsetzen, damit ,wenigstens ein kleiner Schritt zur Linderung der Armut gesetzt wird“'; OTSo044 II 09.08.2008, "Cernic: Mindestsicherung nicht für Wahlkampf-Propaganda missbrauchen, Utl.: Die Kärntner Sozialreferentin fordert den Kärntner Landeshauptmann auf, seine ,Störenfriedposition aufzugeben und die Mindestsicherung nicht zu blockieren“. nur mehr fallweise angestoßen ${ }^{33}$ und die Grünen signalisierten darüber hinaus, dass sie sich mit der "Mindestsicherung von Buchinger anfreunden" können. ${ }^{34}$

In der spätherbstlichen Neuauflage der Großen Koalition besetzte weiterhin die SPÖ das Sozialministerium, allerdings wurde der bisherige Minister Erwin Buchinger von Rudolf Hundstorfer abgelöst, der zuvor Präsident des ÖGB gewesen war. Zwar enthielt das Regierungsprogramm die Absichtserklärung, die Umsetzung der Mindestsicherung „auf Basis der vorliegenden Arbeiten [...] zügig voran[zu]treiben" (Bundeskanzleramt 2008, 188). Tatsächlich folgte jedoch eine Serie weiterer Verhandlungen, sodass die Beschlussfassung über die gegenständliche Art.-I5a-Vereinbarung auf Bundesebene erst in der Nationalratssitzung vom 7. Juli 2010 stattfand.

In diesem Prozess wurde das avisierte Leistungsniveau der Mindestsicherung reduziert. Während das BZÖ-regierte Land Kärnten im Jahr 2009 weiterhin seine Zustimmung zum Mindestsicherungspaket verweigerte, ${ }^{35}$ wurde im Anschluss an den so genannten „Sommerministerrat" der Bundesregierung ein zwischen SPÖ und ÖVP abgestimmter modifizierter Reformplan präsentiert. Nunmehr waren statt der I4 jährlichen Zahlungen nur noch mindestens zwölf jährliche Zahlungen vorgesehen (die Höhe der monatlichen Leistung wurde nicht angehoben). Weiters wurde als Termin für die Umsetzung der Mindestsicherung auf Landesebene statt Jänner 2OIO nunmehr der September 20IO genannt. ${ }^{36}$

Die Leistungsminderung um knapp I5 Prozent ging auf eine Forderung der ÖVP zurück, welche SPÖ-Sozialminister Hundstorfer im Gegenzug für eine (neuerliche) Zusage, die Reform mitzutragen, akzeptierte. ${ }^{37}$ Die ÖVP argumentierte, dass „keine Analogie zum I3. und I4.

33 Vgl. z.B. die folgenden APA- und OTS-Presseaussendungen: OTSoI96 II 26.08.2008, „LIF-Zach fordert endlich echte und faire Grundsicherung, Utl:: Buchinger-Haider-Annäherung bedeutet halbherzigen und unzureichenden Kompromiss - LIF fordert bedingungslose Grundsicherung für soziale Gerechtigkeit“; APA0683 II I8.09.2008, „NR-Wahl - Gemächliches TV-Duell Molterer - Van der Bellen I, Utl.: Unaufgeregte Diskussion über Pensionen, Grundsicherung, Bildung“.

34 Vgl. APA-Presseaussendung: APAo243 II I6.09.2008, „NR-Wahl: Grüne wollen 900 Euro Grundsicherung zwölfmal im Jahr. Utl.: Sozialsprecher Öllinger: Können uns aber auch mit der Mindestsicherung von Buchinger anfreunden".

35 Vgl. APA-Presseaussendung: APAo243 II 16.09.2008, „NR-Wahl: Grüne wollen 900 Euro Grundsicherung zwölfmal im Jahr. Utl.: Sozialsprecher Öllinger: Können uns aber auch mit der Mindestsicherung von Buchinger anfreunden".

36 Vgl. APA-Presseaussendung: APA0385 II 28.07.2009, „Mindestsicherung verschoben und gekürzt, Utl.: Einheitliche Sozialhilfe auf September 2010 verschoben - Regierung will nur noch zwölf statt I4 Monatsraten auszahlen - SP-interner Widerstand gegen, Schmalspurvariante ${ }^{\text {"i. }}$

37 Vgl. APA-Presseaussendung: APAo247 II 28.07.2009, „Mindestsicherung - Hundstorfer verteidigt ,Kompromiss', Utl.: Pröll will Einigung mit allen Bundesländern“. 
Monatsgehalt geschaffen ${ }^{438}$ sowie dass - so der damalige ÖVP-Vizekanzler und Finanzminister Josef Pröll - keine „soziale Hängematte“ geknüpft werden sollte. ${ }^{39}$ Soziale NGOs, die Grünen und sozialdemokratische LandessozialrätInnen kritisierten diese Änderung. ${ }^{40}$ Letztere konnten sich vor dem Hintergrund des Konsenses von SPÖ und ÖVP auf Bundesebene und des Widerstandes des ÖVP-regierten Landes Niederösterreich, der in Folge erneut zu Tage trat ${ }^{41}$ jedoch nicht durchsetzen (Pfeil/ Otter 2OII).

In dieser letzten Phase des Reformprozesses wandelte sich der Kern der inhaltlichen Debatte zusehends, wobei der ursprüngliche armutspolitische Fokus stufenweise durch andere Themen überlagert wurde. Die ÖVP forderte, dass "Leistungsgerechtigkeit“ wieder stärker betont und in der Sozialpolitik das "Gießkannenprinzip" hinterfragt werden müsse. ${ }^{42}$ Das Transfersystem zeige, so die ÖVP mit Bezug auf die sogenannte Prettenthaler-Studie (Prettenthaler/Sterner 2009), zusammen mit dem Steuersystem eine „Schwellenproblematik“ und „Anreizfallen für Berufstätige “43. Die ÖVP setzte zum Jahreswechsel 2009/2010 obendrein das Junktim, dass sie der Mindestsicherung nur zustimmen werde, wenn ein „Transferkonto“ in Form eines personenbezogenen Einkommens- und Leistungsbezugskontos geschaffen werde, da, so die ÖVP, „dem Missbrauch der Mindestsicherung sowie anderer staatlicher Leistungen $[\ldots]$ ein Riegel vorgeschoben ${ }^{4} 44$ werden müsse. SPÖ und ÖVP einigten sich im März 2010 auf eine

38 Der Standard, 28.7.2009, „Nur I2 statt I4 Zahlungen im Jahr“. 39 Der Standard, 4.8.2009, „Handaufhalten in der Hängematte“.

40 Vgl.z.B.folgendeAPA-und OTS-Presseaussendungen:OTSoogo II,CI 28.07.2009, „Weitere Verschlechterungen in der Mindestsicherung: Arme müssen zahlen, Utl.: Armutskonferenz an Finanzministerium: ,Was soll noch alles wegverhandelt werden?'“; OTSoo96 II, CI 28.07.2009, „Öllinger zu Ministerrat: Mindestsicherung zu Grabe getragen, Utl.: ÖVP agiert bei Sozialversicherung nur im Interesse der Versicherungsunternehmen“; APA034I II 28.07.2009, „Mindestsicherung - SPÖ-Landespolitiker lehnen Kürzung ab, Utl.: Flecker: ,Geradezu unerträglich' - auch Oberösterreich und Salzburg dagegen - Sondersitzung der Länderreferenten“.

4I Vgl. OTS-Presseaussendung: OTSoo25 II 08.09.2009, „MiklLeitner: SP-Scharer kolportiert falsches Ergebnis der Sozialreferentenkonferenz, Utl:: I2-malige Auszahlung wurde bereits beschlossen und soll bleiben".

$42 \mathrm{Vgl}$ z.B. APA-Presseaussendung: „ÖVP will schlankeres Programm und mehr soziale Treffsicherheit, Utl.: ÖVP startet Programmdebatte - Kaltenegger für mehr ,Leistungsgerechtigkeit': ,Gießkannenprinzip bei Sozialleistungen hinterfragen - ,Müssen sparen'."

43 Vgl. OTS Pressenaussendung: OTSo24I II 17.II.2009, „Kopf: Leistungsbereitschaft erhalten, Leistungsgerechtigkeit schaffen; Parlament/ÖVP/Klubklausur/PK Kopf "

44 Vgl. OTS Presseaussendung: OTSo286 II, WI, CI 28.06.2010, „Kopf zu Transparenzdatenbank: Missbrauch der Mindestsicherung Riegel vorgeschoben“.
„Transparenzdatenbank" ${ }^{45}$, deren konkreter Inhalt jedoch Gegenstand weiterer Debatten blieb. ${ }^{46}$ Im Übrigen lenkten auch die FPÖ und das BZÖ im Jahr 2010 die Diskussion vermehrt auf Fragen der „Missbrauchsvermeidung" und "Leistungsgerechtigkeit“.47 Die Diskussion kulminierte im September 2010 im Rahmen des Wahlkampfes zum Wiener Landtag, und damit nach der Nationalratsabstimmung zur Art-I5a-B-VG-Vereinbarung, in einer von der ÖVP initiierten breiten politischen Diskussion zur Option, MindestsicherungsbezieherInnen zu gemeinnütziger Arbeit zu verpflichten. In der Diktion der ÖVP sollte dies als „Keule gegen sozialen Missbrauch ${ }^{48}$ wirken, was die FPÖ ${ }^{49}$ unterstützte. Die SPÖ lehnte die Verpflichtung zu gemeinnütziger Arbeit $a b$ und verwies wie in den Monaten davor ${ }^{50}$ darauf, dass die Mindestsicherung ohnehin mit Arbeitsanreizen und Sanktionsmöglichkeiten verknüpft sei um Menschen durch den Vollzug des AMS wieder in den ersten Arbeitsmarkt zu vermitteln. ${ }^{\text {I }}$

Insgesamt verlor nach dem Nationalratswahlkampf 2008 die armutssichernde Funktion der Mindestsicherung in der gegenständlichen politischen Debatte insgesamt zusehends an Bedeutung. Vielmehr traten

45 Die tatsächliche Entwicklung dieser Transparenzdatenbank gestaltete sich in der Folge sehr schleppend. Ende 2015 war eine Erfassung der Leistungen von Ländern und Gemeinden noch nicht absehbar; vgl. APA Presseaussendung: APA0050 II, WI 09.07.2015, „Einbeziehung der Länder in Transparenzdatenbank verzögert sich weiter, Ut1.: Schelling: Kosten-Nutzen-Analyse der Länder nicht vor Herbst fertig, danach Verhandlungen".

46 Vgl. APA-Presseaussendungen: APA0514 II 28.06.2010, „Mindestsicherung und Transparenzdatenbank - Eine Chronologie";

47 Vgl. z.B. die folgenden APA- und OTS-Presseaussendungen: OTSo226 II, CI OI.09.2010, „BZÖ-Ebner: Mindestsicherung ist leistungsfeindlich und Hängemattensozialismus in Reinkultur“; OTSo23I II, CI 07.07.20I0, „BZÖ-Bucher: Mindestsicherung ist Hängemattensozialismus á la DDR, Utl.: Alle fleißig arbeitenden Österreicherinnen und Österreicher werden benachteiligt"; OTSo227 II, 07.07.20IO, „Kickl: Hundstorfer versetzt mit Mindestsicherung der arbeitenden Bevölkerung einen ,Schlag ins Gesicht', Utl.: Gesetz über einen entsprechenden Mindestlohn fehlt noch immer".

48 So die damalige Familienstaatssekretärin Christine Marek; vgl. APA Presseaussendung: APAo047 II, WI I4.09.2010, „Mindestsicherung: ÖVP für Arbeitspflicht nach sechs Monaten, Utl.: Marek: ,Keule gegen sozialen Missbrauch'“.

49 Vgl. OTS-Presseaussendung: OTSoI59 II, I5.09.2010, „FPÖ-Kickl: Werden ÖVP in Sachen Mindestsicherungsmissbrauch testen, Utl.: FPÖ bringt Antrag für Regierungsvorlage ein - neugierig auf Abstimmungsverhalten der ÖVP-Mandatare“.

5o Vgl. z.B. die folgenden OTS-Presseaussendungen: OTSoI54 II, 05.02.2009, „Hundstorfer: ,Bedarfsorientierte Mindestsicherung ist keine soziale Hängematte ““; OTSOI52 CI, II O2.03.2010, „Hundstorfer: Weg frei für Mindestsicherung Utl.: Transparenzdatenbank schafft Überblick aller staatlichen Leistungen"; OTSO255 II, 25.05.20IO, „Hundstorfer: Beschäftigung ist das wirkungsvollste Instrument gegen Armut und soziale Ausgrenzung"; vgl. z.B. auch: Der Standard, Interview mit Sozialminister Hundstorfer, „Auf den Häkel mit der ÖVP gehe ich keine Sekunde ein".

5I Vgl. APA-Presseaussendung: APAoI49 II, WI I4.09.20IO, "Mindestsicherung: ÖVP-Vorstoß für SPÖ „billiger Wahlkampfgag“, Utl.: Hundstorfer verweist auf jetzt schon vorhandene Sanktionen Diskutieren Marek-Vorschlag, sicher nicht'“. 
Themenbereiche wie „Sozialmissbrauch“, „Leistungsgerechtigkeit" und die Anbindung an den Arbeitsmarkt als Problemframing in den Mittelpunkt der Auseinandersetzung zwischen den politischen Parteien. In anderen Worten: Zuerst stand die Herstellung einer österreichweiten vereinheitlichten und armutsfesten Leistung im Zentrum, dann verschob sich der Fokus auf die ReIntegration in den Arbeitsmarkt, was im Vergleich zu den ursprünglichen Plänen unter anderem mit Leistungseinschränkungen einherging. Ungeachtet dessen wurde die Bedarfsorientierte Mindestsicherung jedoch auf internationaler Ebene, insbesondere gegenüber der Europäischen Kommission, weiterhin als österreichweite armutsmindernde "Panakeia“ präsentiert (vgl. z.B. Bundeskanzleramt 2013, 33f.).

\section{Diskussion und Schlussfolgerungen}

Vom Agendasetting bis zur Beschlussfassung der Reform der Sozialhilfe in Form der Bedarfsorientierten Mindestsicherung vergingen mehr als zehn Jahre. Es zeigt sich, dass die gegenständliche Entwicklung nur dann verstanden werden kann, wenn mehrere erklärende Faktoren berücksichtigt werden. Dabei sind die von verschiedenen Theorieschulen forcierten Argumente nicht wechselseitig ausschließend, sondern in fruchtbarer Weise wechselseitig anschlussfähig. Ein monokausaler Versuch der Begründung würde hingegen aufgrund der komplexen Bedingungen wichtige Aspekte außer Acht lassen.

Trotzdem kristallisiert sich ein wichtiger Faktor heraus, nämlich jener der Parteiendifferenz. Die inhaltlichen Entwicklungen und Veränderungen im Zeitverlauf können primär mit Bezug auf die Strategien und Positionierungen der Sozialdemokratie erklärt werden, die im gegenständlichen Reformprozess den wegweisenden Akteur darstellt. Wie sich die SPÖ jedoch in der langjährigen Debatte - und damit in der mehr oder minder starken (und wandelnden) Vertretung von Interessen von Outsidern positionierte - wurde wesentlich von einer Reihe weiterer Kontextfaktoren geprägt. Verallgemeinernde Thesen über die wahrscheinliche Positionierung sozialdemokratischer Parteien im Bereich Mindestsicherung, wie sie sich im Gros der einschlägigen Literatur finden, greifen für sich genommen hingegen zu kurz.

In der ersten Phase betrieb die SPÖ das Thema der Mindestsicherung aus einer Oppositionsrolle heraus. In Abgrenzung zur bestehenden Mitte-rechts-Regierung verfolgte sie das Ziel, ihr soziales Profil zu schärfen. Die vorgeschlagene Reform der Sozialhilfe - mit der expliziten Forderung der Armutsminderung - war Teil einer breiteren, zu diesem Zweck formulierten Programmatik. Die Positionierung der SPÖ - in direkter Konkurrenz zu einer Mitte-rechts-Regierung - für so genannte Out- sider entspricht nicht den gängigen Erklärungsansätzen. Diese würden sozialdemokratische Parteien eher eine Vertretung von Insidern erwarten, insbesondere wenn sie mit christlich-sozialen Parteien im Wettbewerb stünden. Einer der Erklärungsgründe könnte darin liegen, dass die SPÖ mit der Mindestsicherung ein Thema mitbesetzte, das schon länger auf der Agenda anderer sozial progressiver bzw. liberaler Parteien (Grüne und LIF) stand. Zudem nutzte die SPÖ das schon vorher durch soziale NGOs und WissenschafterInnen vorgenommene Problemframing, bei dem die Sozialhilfe als nur rudimentäre Form der Armutssicherung breit kritisiert wurde.

Als unterstützender Faktor wirkte beim Agendasetting zudem, dass die Bundesländer und die SoziallandesrätInnen einen Reformbedarf wahrnahmen, dessen Ausgangspunkt die steigenden finanziellen Belastungen der Sozialhilfe bildeten. Die Forcierung des Themas Mindestsicherung innerhalb der SPÖ wurde weiters unter anderem dadurch ermöglicht, dass die programmatische Positionierung der Partei im Vorfeld des Wahlkampfes 2006 an kleine Kompetenzteams übertragen wurde. In jenem für Soziales stellten damalige SoziallandesrätInnen eine treibende Kraft dar. Neben der sozialpolitischen Motivation, eine Verbesserung des Leistungsniveaus und des Zugangs für Betroffene zu erwirken, dürften für sie auch unmittelbare Interessen der Bundesländer handlungsleitend gewesen sein. Letzteres in dem Sinn, dass die vorgeschlagene Reform durch einen besseren Sicherungsstandard im vorgelagerten Sozialversicherungssystem und eine partielle Kostenübernahme der Krankenversicherungskosten durch den Bund eine Eindämmung von Kosten(zuwächsen) bei den Ländern erwarten ließ. Dass auch die Gewerkschaften als weiterer zentraler und eng mit der SPÖ verwobener sozialpolitischer Akteur den gegenständlichen Reformvorschlag mittrugen bzw. trotz einer grundsätzlich eher kritischen Position zu bedarfsgeprüften Leistungen nicht gegen diesen opponierten, dürfte unter anderem darauf zurückgehen, dass zugleich mit der Mindestsicherungsreform eine partielle Erhöhung von Leistungen aus der Arbeitslosenversicherung, konkret in Form der Notstandshilfe, geplant war.

Ein weiteres zentrales Element war innerparteilich und im Wechselspiel mit anderen AkteurInnen die Rolle Erwin Buchingers als policy-entrepreneur, der einer armutspolitischen Prämisse folgend für eine Reform der Sozialhilfe eintrat. Erwin Buchinger war als damaliger Salzburger Soziallandesrat Mitglied des angesprochenen SPÖ-internen Kompetenzteams, verhandelte das Thema "Soziales" mit dem späteren Regierungspartner ÖVP und stieg daraufhin zum Sozialminister auf.

Insgesamt waren damit kontextspezifische Faktoren und personelle Konstellationen dafür zentral, dass die SPÖ das Thema Mindestsicherung auf ihre Agenda 
setzte und explizit als armutspolitisches Instrument forcierte. Gleichzeitig wurde für eine relativ breite Koalition von AkteurInnen - namentlich der SPÖ, der Gewerkschaften, der Bundesländer und, mit inhaltlichen Abweichungen, auch der Grünen, des LIF und sozialer NGOs - die gemeinsame Idee zentral, dass die Sozialhilfe in Richtung einer bedarfsgerechten Mindestsicherung reformiert werden sollte, die soziale Exklusion im umfassenden Sinn verhindert. Und die Dominanz dieser Idee machte es für die ÖVP (vorerst) offensichtlich schwierig, offen gegen dieselbe zu opponieren.

Die Beschlussfassung der ursprünglich geplanten Reform gelang jedoch trotz der Existenz dieser Allianz und trotz der starken Rolle eines zentralen politischen Entrepreneurs nur mit substantiellen Abstrichen. Dies ist einerseits der gegebenen Akteurskonstellation geschuldet, die sich durch eine Vielzahl an Vetopunkten bzw. Vetospielern auszeichnet. Opponierte nur ein Akteur gegen ein bestimmtes Element des Reformplanes - sei es aus wahlstrategischen, inhaltlichen oder budgetären Gründen - so wurden die politischen Aushandlungen verzögert und ursprüngliche Reformpläne häufig zugunsten einer weitgehenden Beibehaltung des Status Quo ad acta gelegt. So führten etwa Widerstände der Länder und des AMS bereits in einer frühen Phase der Verhandlungen dazu, dass der ursprünglich vorgesehene breite institutionelle Umbau im Sinn eines One-StopShops rasch verworfen wurde. Andererseits wird der nur begrenzte innerparteiliche Konsens innerhalb der SPÖ betreffend eine - im umfassenderen Sinn - verbesserte soziale Absicherung von Outsidern bereits hier deutlich, indem diesbezüglich nicht auf einen Pfadwechsel insistiert wurde.

Dieses Bild verdichtet sich ab 2008 vor dem Hintergrund weiterer veränderter Rahmenbedingungen. Mit dem Abgang von Erwin Buchinger ging der zuvor treibende policy-entrepreneur verloren. Zudem erhöhte sich vor dem Hintergrund der sogenannten Finanz- und Wirtschaftskrise der budgetpolitische Druck, was es für die SPÖ offensichtlich zunehmend weniger opportun erscheinen ließ, für höhere Ausgaben für so genannte Outsider einzutreten. In ähnlicher Weise gewannen bei den Bundesländern budgetpolitische Motive an Bedeutung. Darüber hinaus forcierten nun der Koalitionspartner der SPÖ, die ÖVP, sowie die (in Ansätzen) bereits wieder erstarkte FPÖ selbst das Thema Mindestsicherung, allerdings mit Fokus auf Fragen nach „Leistungsgerechtigkeit" und "Sozialmissbrauch".

Im Zuge dieser Entwicklungen ging die Leitidee, dass eine Reform der Sozialhilfe in Richtung einer bedarfsgerechten Mindestsicherung darauf abzielen sollte, soziale Ausgrenzung im umfassenden Sinn zu verhindern, zunehmend verloren. Im letzten Drittel der Verhandlungen repositionierte sich dabei die SPÖ dahingehend, dass sie nun nicht mehr vom Ziel einer einheitlichen armutsfesten Leistung sprach, im Vergleich zu den ursprünglichen Plänen substantielle Leistungsreduktionen akzeptierte, und das Reformvorhaben nun vor allem mit der intensiveren Betreuung der BezieherInnen beim AMS begründete.

Am österreichischen Fall wird insgesamt ersichtlich, dass sozialdemokratische Parteien - unter bestimmten "günstigen“ Rahmenbedingungen und mobilisiert von individuellen AkteurInnen - auch die Interessen von Outsidern vertreten. Allerdings offenbart sich, dass eine nachhaltige Vertretung dieser Interessen eher unwahrscheinlich ist. Ändert sich der Kontext in eine Richtung, die ein solches Engagement aus strategischen Überlegungen heraus fragwürdig erscheinen lässt, z.B. vor dem Hintergrund einer durch konservative und Rechtsparteien betriebenen "Sozialmissbrauchs"-Debatte oder aufgrund eines starken Spardrucks, der andernfalls zu Einschnitten für Insider führen könnte, so besteht eine hohe Bereitschaft, vom ursprünglichen Kurs bzw. der früheren Argumentationsbasis abzurücken. ${ }^{52}$ Das in der Literatur beschriebene Argument einer stärkeren Positionierung gegenüber anderen linken Parteien wurde über weite Strecken durch den politischen Wettbewerb mit der ÖVP und der FPÖ überlagert, denen es im Endeffekt gelang, den inhaltlichen Fokus der gesamten politischen und öffentlichen Debatte zu verschieben. Schlussendlich war damit die Frage, ob und wie konservative und rechts positionierte politische Kräfte das Thema der Mindestsicherung aufgreifen, für den Ausgang der Reform zentraler als die Rolle von anderen betreffend der Mindestsicherung sozialpolitisch progressiven Parteien (Grüne und LIF).

In diesem Beitrag wurde vor allem die Rolle der SPÖ analysiert, nicht jedoch näher dem Wandel der Positionierung der ÖVP nachgegangen. Wenngleich in der einschlägigen Literatur allgemein subsumiert wird, dass christlich-konservative Parteien in der Regel gegen eine verstärkte Absicherung von Armutsgefährdeten durch Mindestsicherungssysteme eintreten, könnten die Konzessionen der SPÖ hier ursprünglich einen stärkeren Widerstand der ÖVP in den Koalitionsverhandlungen verhindert haben. Weitere Forschungen sollten ebenso die Rolle der sozialdemokratischen und christlichen Gewerkschaften, von karitativen Organisationen sowie die Machtverhältnisse und Auseinandersetzungen zwischen Bund und Länder näher beleuchten. Ein weiterer möglicher relevanter Erklärungsfaktor sind Wahlen auf Landesebene.

52 Im gegenständlichen Beispiel erfolgte dies im Übrigen vor dem Hintergrund, dass es an einer breiten innerparteilichen Verankerung des Themas mangelte, was bereits im innerparteilichen Agendasetting seinen Ausgang hatte (aber dieses unter Umständen zugleich überhaupt erst ermöglichte). 


\section{Ausblick}

Die Art.-I5a-B-VG-Vereinbarung zur Mindestsicherung lief 20I6/17 aus und wurde in Folge nicht mehr verlängert. Hintergrund war, dass einzelne Bundesländer Leistungsniveaus nach unten nivellieren wollten bzw. dies bereits zuvor (in Verletzung der damals noch geltenden Art.-I5a-B-VG-Vereinbarung) getan hatten, was vom damaligen SPÖ-Sozialminister und auch vom Bundesland Wien, wo es mit Abstand die höchste Quote an MindestsicherungsbezieherInnen gibt, abgelehnt wurde. Die Ende 2017 angelobte neue Bundesregierung aus ÖVP und FPÖ kündigte in ihrem Regierungsprogramm bzw. in einem Ministerratsvortrag von Ende Mai 2018 an, die Mindestsicherung durch ein Bundesrahmengesetz neu zu regeln. Inhaltlich soll dabei vor allem der Leistungszugang für Personen mit Migrationshintergrund erschwert bzw. zum Teil unmöglich gemacht werden, und im Vergleich zu den früheren Mindeststandards gemäß der Art.-I5a-B-VG-Vereinbarung sollen Leistungen durch festgelegte Maximalsätze zum Teil substantiell gekürzt werden (vgl. Fink 20I8). Diese Entwicklung vollzieht sich einerseits vor dem Hintergrund, dass ein zentraler Effekt der oben beschriebenen Transformation der Sozialhilfe unter dem Begriff „Mindestsicherung“ retrospektiv betrachtet die Zerstörung des Momentums des früheren durch die sozialen NGOs und WissenschafterInnen (ebenso durch die Grünen und das LIF) unter dieser Überschrift etablierten Diskurses war, der in erster Linie auf eine breite Armutsminderung abzielte. An seine Stelle trat andererseits vermehrt eine Debatte über "Leistungsgerechtigkeit“, „Arbeitsanreize“ und „Sozialmissbrauch“, die vor allem von ÖVP und FPÖ forciert und in den letzten Jahren häufig mit Blick auf Personen mit Migrationshintergrund zugespitzt wurde. Letzteres trug wohl wesentlich dazu dabei, dass es ÖVP und FPÖ gelang, Fragen der Armutsminderung fast vollständig aus öffentlich dominanten Problemrahmungen im Kontext von Mindestsicherung zu verdrängen. Eine genauere Untersuchung diesbezüglicher Zusammenhänge ist jedoch der weiteren Forschung zum Thema vorbehalten.

\section{Literatur}

Anderson, Christopher J./Pablo Beramendi (2012), Left Parties, Poor Voters, and Electoral Participation in Advanced Industrial Societies, in: Comparative Political Studies, Vol. 45, 7 I4-746.

Bahle, Thomas/Vanessa Hubl/Michaela Pfeifer (2OII), The Last Safety Net: A Handbook of Minimum Income Protection in Europe, Bristol: Policy Press.

Béland, Daniel (2005), Ideas and Social Policy: An Institutionalist Perspective, in: Social Policy \& Administration, Vol. 39, I-I8.
Béland, Daniel/Robert Henry Cox (20I6), Ideas as coalition magnets: coalition building, policy entrepreneurs, and power relations, in: Journal of European $\mathrm{Pu}$ blic Policy, Vol. 23, 428-445.

BMSK (Bundesministerium für Soziales und Konsumentenschutz) (2009). Sozialbericht 2007 - 2008: Ressortaktivitäten und sozialpolitische Analysen, Wien.

Bock-Schappelwein, Julia (2004), Arbeitslosigkeit und offene Sozialhilfe in Österreich: Eine quantitative Analyse, Wien.

Bonoli, Giuliano (2007), Time Matters: Postindustrialization, New Social Risks, and Welfare State Adaptation in Advanced Industrial Democracies, in: Comparative Political Studies, Vol. 40, 495-520.

Bonoli, Giuliano/Cyrielle Champion (2015), Federalism and Welfare to Work in Switzerland: The Development of Active Social Policies in a Fragmented Welfare State, in: Publius: The Journal of Federalism, Vol. 45, 77-98.

Bothfeld, Silke/Sigrid Betzelt (2013), How Do Activation policies Affect Social Citizenship? The Issue of Autonomy, in: Ramia, Gaby/ Kevin Farnsworth/Zöe Irving (Hg.), Social Policy Review: Analysis and Debate in Social Policy, Bristol/Chicago: Policy Press, 249-270.

Buchinger, Erwin (2006), Arbeit. Chancen. Sicherheit: Die bedarfsorientierte Grundsicherung - Weg aus der Armut: Die bedarfsorientierte Grundsicherung, Version 3, Stand OI.I2.2006, Wien.

Buchinger, Erwin (2008), Eingangsstatement, in: BMSK (Hg.), Bedarfsorientierte Mindestsicherung: Dokumentation der Fachtagung vom II. Dezember 2007, Wien, 7-8.

Bundeskanzleramt (2000), Österreich neu regieren: Regierungsprogramm der Österreichischen Bundesregierung für die XXI. Gesetzgebungsperiode, Wien.

Bundeskanzleramt (2003), Regierungsprogramm der Österreichischen Bundesregierung für die XXII. Gesetzgebungsperiode, Wien.

Bundeskanzleramt (2007), Regierungsprogramm für die XXIII. Gesetzgebungsperiode: 2007-20IO, Wien.

Bundeskanzleramt (2013), Nationales Reformprogramm Österreich 20I3, Wien, Internet: http://archiv.bundeskanzleramt.at/DocView.axd?CobId=5II22 (Zugriff: oI.03.2015)

Clasen, Jochen/ Daniel Clegg (20II), Regulating the Risk of Unemployment: National Adaptations to PostIndustrial Labour Markets in Europe, Oxford/New York: Oxford University Press.

Clasen, Jochen/ Daniel Clegg (2012), Adapting Labour Market Policy to a Transformed Employment Structure: The Politics of 'Triple Integration', in: Giuliano Bonoli/David Natali(Hg.), The Politics of the New Welfare State, Oxford: Oxford University Press, I35-I57.

Clegg, Daniel (2014), Convergence from Below? The Reform of Minimum Income Protection in France and 
the UK, in: Journal of International and Comparative Social Policy, Vol. 30, I47-I64.

Collier, David (20II), Understanding process tracing, in: PS: Political Science \& Politics, Vol. 44, 823-830.

Die Grünen (1998), Die Grüne Grundsicherung: Wir geben Sicherheit. Wir sichern Chancen, Wien.

Dimmel, Nikolaus (2008), Bedarfsorientierte Mindestsicherung - ein Meilenstein?, in: WISO, Vol. 3I: 30-47.

Ebbinghaus, Bernhard (2006), Trade Union movements in post-industrial welfare states: Opening up to new social interests?, in: Klaus Armingeon/Giuliano Bonoli (Hg.), The Politics of Post-Industrial Welfare States: Adapting post-war social policies to new social risks, London: Routledge, I23-I43.

Emmenegger, Peter/Silja Häusermann/Bruno Palier/Martin Seeleib-Kaiser (2012). The Age of Dualization: The Changing Face of Inequality in Deindustrializing Societies, Oxford/New York: Oxford University Press.

Esping-Andersen, Gøsta (1990), The Three Worlds of Welfare Capitalism, Cambridge: Polity Press.

Ferrera, Maurizio (I993), Modelli di Solidarietà: Politica e Riforme Sociali nelle Democrazie, Bologna : Il Mulino.

Figari, Francesco/Manos Matsaganis/Holly Sutherland (2013), Are European Social Safety Nets Tight Enough? Coverage and Adequacy of Minimum Income Schemes in I4 EU Countries, in: International Journal of Social Welfare, Vol. 22, 3-I4.

Fink, Marcel (2006), Zwischen „Beschäftigungsrekord“ und "Rekordarbeitslosigkeit": Arbeitsmarkt und Arbeitsmarktpolitik unter Schwarz-Blau/Orange, in: Emmerich Tálos (Hg.), Schwarz-Blau: Eine Bilanz des "Neu-Regierens", Wien/Münster: LIT, I7O-I87.

Fink, Marcel (2018), Austria: Government reform plan for Minimum Income Scheme causes ongoing debates, ESPN Flash Report 20I8/47, July 20I8, Internet: http://ec.europa.eu/social/BlobServlet?docId=I9975 \&langId=en (Zugriff: I8.09.2018)

Flecker, Jörg/Christoph Hermann (2009), Das österreichische Beschäftigungsmodell im Spiegel von Branchenanalysen, in: Christoph Hermann/Roland Atzmüller (Hg.), Die Dynamik des „österreichischen Modells": Brüche und Kontinuitäten im Beschäftigungsund Sozialsystem, Berlin: Sigma, 73-9I.

Flora, Peter/Jens Alber (I98I), Modernization, Democratization, and the Development of Welfare States in Western Europe, in: Peter Flora/Arnold Heidenheimer (Hg.), The Development of Welfare States in Europe and America, New Brunswick/London: Transaction Books, 37-8o.

George, Alexander L./Andrew Bennett (2005), Case Studies and Theory Development in the Social Sciences, Cambridge/London: MIT Press.

Gough, Ian/Jonathan Bradshaw/John Ditch/Tony Eardley/ Peter Whiteford (1997), Social Assistance in OECD
Countries, in: Journal of European Social Policy, Vol. 7, I7-43.

Green-Pedersen, Christoffer (2002), The Politics of Justification: Party Competition and Welfare-State Retrenchment in Denmark and the Netherlands from 1982-I995, Amsterdam: Amsterdam University Press.

Guy Peters, Brainard (20II), Approaches in comparative politics, in: Daniele Caramini (Hg.), Comparative Politics, Oxford/New York: Oxford University Press, 37-49.

Haberbauer Judith/Hans Steiner/Agnes Streissler (1999), Einbinden statt Ausgrenzen: Neue Strategien gegen die Armut, Bericht einer ExpertInnenarbeitsgruppe, Wien.

Hall, Peter A. (I993), Policy Paradigms, Social Learning, and the State: The Case of Economic Policymaking in Britain, in: Comparative Politics, Vol. 25, 275-296.

Hay, Colin (2004), Ideas, Interests and Institutions in the Comparative Political Economy of Great Transformations, in: Review of International Political Economy, Vol. II, 204-226.

Häusermann, Silja/Georg Picot/Dominik Geering (2010), Rethinking Party Politics and the Welfare State: Recent Advances in the Literature, Paper prepared for the I7th International Conference of the Council for European Studies, Montréal, April I5-I7, 2010.

Hermann, Christoph/ Jörg Flecker (2009), Betriebliche Interessenvertretung in Österreich. Wachsender Druck und zunehmende Lücken, in: Hermann, Christoph/ Roland Atzmüller (Hg.), Die Dynamik des „österreichischen Modells“: Brüche und Kontinuitäten im Beschäftigungs- und Sozialsystem, Berlin: Sigma, 93-IIO.

Hibbs, Douglas A. (1977), Political Parties and Macroeconomic Policy, in: The American Political Science Review, Vol. 7I, I467-I487.

Huber, Evelyne/John D. Stephens (2000), Partisan Governance, Women's Employment, and the Social Democratic Service State, in: American Sociological Review, Vol. 65, 323-342.

Iversen, Torben/David Soskice (2009), Dualism and political coalitions: Inclusionary versus exclusionary reforms in an age of rising inequality, Paper prepared for presentation at the Annual Meeting of the American Political Science Association, Toronto, September 3-6, 2009.

Jessoula, Matteo/Julia Kubisa/Ilaria Madama/Marianna Zielenska (2014), Understanding convergence and divergence: old and new cleavages in the politics of minimum income schemes in Italy and Poland, in: Journal of International and Comparative Social Policy, Vol. 3O(2), I28-I 46. 
Kersbergen van, Kees/Kahlyvas Stahis (2010), Christian Democracy, in: Annual Reviews of Political Science, Vol. I3, I83-209.

King, Desmond/David Rueda (2008), Cheap Labor: The New Politics of "Bread and Roses" in Industrial Democracies, in: Perspectives on Politics, Vol. 6, 279-297.

Kingdon, John W. (1984), Agendas, Alternatives, and Public Policies, Boston: Little Brown.

Köppl, Franz/Hans Steiner (I989), Sozialhilfe - ein geeignetes Instrument zur Bekämpfung sozialer Not?, in: Kammer für Arbeiter und Angestellte für Wien (Hg.), Sozialhilfe: Strukturen, Mängel, Vorschläge, Wien: Verlag des Österreichischen Gewerkschaftsbundes, 33-IIO.

Korpi, Walter (I983), The Democratic Class Struggle, Boston: Verlag.

Leibetseder, Bettina/Iris Woltran (2OII), Aktivierung: Ein zentrales Element in der Sozialhilfe und Bedarfsorientierten Mindestsicherung, in: Pfeil, Walter J./Josef Wöss (Hg.), Bedarfsorientierte Mindestsicherung, Wien: ÖGB-Verlag, 65-80.

LIF (Liberales Forum) (I997), Beschluss betreffend Grundsicherung, ökologische Steuerreform und allgemeine Steuerreform, Salzburg.

Lijphart, Arend (197I), Comparative Politics and the Comparative Method, in: The American Political Science Review, Vol. 65, 682-693.

Lødemel, Ivar/Amilcar Moreira (20I4), Activation or Workfare? Governance and Neo-Liberal Convergence, New York/Oxford: Oxford University Press.

Madama, Ilaria (20II), Beyond continuity? Italian social assistance policies between institutional opportunities and agency, in: International Journal of Social Welfare, Vol. 22, 58-68.

Madama, Ilaria/Matteo Jessoula/Marcelo Natali (2014), Minimum Income: The Italian Trajectory. One, no one and one hundred thousand minimum income scheme, Ort: Centro Einaudi, Laboratorio di Politica Comparata e Filosofia Pubblica.

Mahoney, James (2007), Qualitative Methodology and Comparative Politics, in: Comparative Political Studies, Vol. 4O, I22-I 44.

Marchal, Sarah/Ive Marx/Natascha van Mechelen (20I4), The Great Wake-Up Call? Social Citizenship and Minimum Income Provisions in Europe in Times of Crisis, in: Journal of Social Policy, Vol. 43(2), 247-267.

Marx, Ive/Kenneth Nelson (2013), A New Dawn for Minimum Income Protection, in: Marx, Ive/Kenneth Nelson (Hg.), Minimum Income in Flux, Basingstoke/ New York: Palgrave Macmillan, 28-53.

Melinz, Gerhard (1996), Von der Armenfürsorge zur Sozialhilfe: Projektendbericht für das BM für Wissenschaft, Verkehr und Kunst.

Natili, Marcello (2014), The crisis and the politics of minimum income protection in Southern Europe: Exp- laining the evolution of regional minimum income schemes in Italy and Spain, Paper prepared for the 2OI4 ISEG Conference "The welfare state in Portugal in the age of austerity", Lisboa, 9 - Io May 2014.

Natili, Marcello (2018), Explaining different trajectories of minimum income schemes: Groups, parties and political exchange in Italy and Spain, in: Journal of European Social Policy, Vol. 28(2), II6-I29.

Nelson, Kenneth (2OI3), Social Assistance and EU Poverty Thresholds 1990-2008: Are European Welfare Systems Providing Just and Fair Protection Against Low Income?, in: European Sociological Review, Vol. 29, 386$4 \mathrm{OI}$.

Obinger, Herbert/Emmerich Tálos (20IO), Janus-faced Developments in a Prototypical Bismarckian Welfare State: Welfare Reforms in Austria Since the I970s, in: Palier, Bruno (Hg.), A long goodbye to Bismarck? the Politics of Welfare Reform in Continental Europe, Amsterdam: Amsterdam University Press, IOI-I28.

Pfeil, Walter J. (2008), Umsetzung der Bedarfsorientierten Mindestsicherung: Aktueller Stand und offene Fragen, in: BMSK (Hg.), Bedarfsorientierte Mindestsicherung: Dokumentation der Fachtagung vom II. Dezember 2007, Wien, 9-I3.

Pfeil, Walter J./ Andrea Otter (2OII), Chronologie der Entwicklung der bedarfsorientierten Mindestsicherung, in: Pfeil, Walter J./ Josef Wöss (Hg.), Bedarfsorientierte Mindestsicherung, Wien: ÖGB-Verlag, 209-22I.

Pierson, Paul (1994), Dismantling the welfare state? Reagan, Thatcher, and the politics of retrenchment, Cambridge: Cambridge University Press.

Pierson, Paul (1995), Fragmented Welfare States: Federal Institutions and the Development of Social Policy, in: Governance, Vol. 8, 449-478.

Pierson, Paul (200I), Coping with Permanent Austerity: Welfare State Restructuring in Affluent Democracies, in: Pierson, Paul (Hg.), The New Politics of the Welfare State, Oxford/ New York: Oxford University Press, 4IO-456.

Prettenthaler, Franz/Cornelia Sterner (2009), Aufgabe Soziale Gerechtigkeit in der Steiermark: Anreiztheoretische Untersuchung zur Wechselwirkung von Gemeinde- bzw. Landestranfers und progressivem Steuersystem. Teilbericht zu Modul 3 des Projektes "Aufgabe Soziale Gerechtigkeit in der Steiermark“ des Dr.-Karl-Kummer-Instituts für Steiermark, Graz.

Ross, Fiona (2000), 'Beyond Left and Right': The New Partisan Politics of Welfare, in: Governance: An International Journal of Policy and Administration, Vol. I3(2), I55-I83.

Rueda, David (2005), InsiderOutsider Politics in Industrialized Democracies: The Challenge to Social Democratic Parties, in: American Political Science Review, Vol. 99, 6I-74. 
Rueda, David (2007), Social Democracy Inside Out: Partisanship and Labor Market Policy in Industrialized Democracies, Oxford/New York: Oxford University Press.

Sabatier, Paul A./Hank C. Jenkins-Smith (Hg.) (1993), Policy Change and Learning: an Advocacy Coalition Approach, Boulder: Westview Press.

Siegel, Nico A. (2002), Baustelle Sozialpolitik: Konsolidierung und Rückbau der Sozialpolitik, Frankfurt am Main/New York: Campus.

Schmidt, Manfred G. (I982), Wohlfahrtsstaatliche Politik unter bürgerlichen und sozialdemokratischen Regierungen: Ein internationaler Vergleich, Frankfurt am Main/ New York: Campus.

Schmidt, Vivien A. (2008), Discursive Institutionalism: The Explanatory Power of Ideas and Discourse, in: Annual Review of Political Science, Vol. II, 303-326.

Tálos, Emmerich (2003), Grundsicherung: Eine alte und aktuelle Debatte, in: Emmerich Tálos (Hg.), Bedarfsorientierte Grundsicherung, Wien: Mandelbaum, I57-I72.

Tálos, Emmerich (2008a), Armutspolitik am Beispiel Österreichs: Bedarfsorientierte Mindestsicherung, in: WSI Mitteilungen, $\mathrm{H}$ 3, I59-I63.

Tálos, Emmerich (2008b), Sozialpartnerschaft: Ein zentraler politischer Gestaltungsfaktor in der Zweiten Republik, Innsbruck/Wien/Bozen: Studienverlag.

Tálos, Emmerich/Ulrike Mühlberger (1999), Atpyische Beschäftigung in Österreich, in: Tálos, Emmerich (Hg.), Atypische Beschäftigung: internationale Trends und sozialstaatliche Regelungen, Wien: Manz, 252-284.

Taylor-Gooby, Peter (2004), New Risks, New Welfare: the Transformation of the European Welfare State, Oxford: Verlag.

Weishaupt, J. Timo (20II), From the Manpower Revolution to the Activation Paradigm: Explaining Institutional Continuity and Change in an Integrating Europe, Amsterdam: DeGruyter.

Wilensky, Harold L./Charles N. Lebeaux (1958), Industrial Society and Social Welfare: the Impact of Industrialization on the Supply and Organization of Social Welfare Services in the United States, New York: The Free Press.

Wohlgenannt, Lieselotte/Herwig Büchele (1985), Grundeinkommen ohne Arbeit. Auf dem Weg zu einer kommunikativen Gesellschaft, Wien: Verlag.

\section{Autoren}

Marcel Fink ist Senior Researcher am Institut für Höhere Studien in Wien. Forschungsfelder: Sozial- und Arbeitsmarktpolitik in Österreich und im internationalen Vergleich, soziale Ungleichheit, Einstellungen zu Sozialpolitik.
Bettina Leibetseder ist Professorin für Politikwissenschaft und Soziale Arbeit an der HAW Landshut. Forschungsfelder: Sozial- und Arbeitsmarktpolitik in Österreich und im internationalen Vergleich, StreetLevel-Bureaucracy.

The authors have declared that no competing interests exist. 